\title{
RESEARCH
}

Open Access

\section{Xylitol enhances synthesis of propionate in the colon via cross-feeding of gut microbiota}

Shasha Xiang ${ }^{1 \dagger}$, Kun Ye ${ }^{1 \dagger}$, Mian Li², Jian Ying ${ }^{3}$, Huanhuan Wang ${ }^{4,5}$, Jianzhong Han', Lihua Shi ${ }^{2}$, Jie Xiao ${ }^{3}$, Yubiao Shen ${ }^{6}$, Xiao Feng ${ }^{1}$, Xuan Bao ${ }^{1}$, Yiqing Zheng ${ }^{1}$, Yin Ge' ${ }^{1}$ Yalin Zhang ${ }^{1}$, Chang Liu', Jie Chen ${ }^{1}$, Yuewen Chen ${ }^{1}$, Shiyi $\operatorname{Tian}^{1}$ and Xuan Zhu ${ }^{1 *}$ (D)

\begin{abstract}
Background: Xylitol, a white or transparent polyol or sugar alcohol, is digestible by colonic microorganisms and promotes the proliferation of beneficial bacteria and the production of short-chain fatty acids (SCFAs), but the mechanism underlying these effects remains unknown. We studied mice fed with $0 \%, 2 \%(2.17 \mathrm{~g} / \mathrm{kg} / \mathrm{day})$, or $5 \%$ $(5.42 \mathrm{~g} / \mathrm{kg} /$ day) (weight/weight) xylitol in their chow for 3 months. In addition to the in vivo digestion experiments in mice, $3 \%$ (weight/volume) $(0.27 \mathrm{~g} / \mathrm{kg} /$ day for a human being) xylitol was added to a colon simulation system (CDMN) for 7 days. We performed 16S rRNA sequencing, beneficial metabolism biomarker quantification, metabolome, and metatranscriptome analyses to investigate the prebiotic mechanism of xylitol. The representative bacteria related to xylitol digestion were selected for single cultivation and co-culture of two and three bacteria to explore the microbial digestion and utilization of xylitol in media with glucose, xylitol, mixed carbon sources, or nocarbon sources. Besides, the mechanisms underlying the shift in the microbial composition and SCFAs were explored in molecular contexts.
\end{abstract}

Results: In both in vivo and in vitro experiments, we found that xylitol did not significantly influence the structure of the gut microbiome. However, it increased all SCFAs, especially propionate in the lumen and butyrate in the mucosa, with a shift in its corresponding bacteria in vitro. Cross-feeding, a relationship in which one organism consumes metabolites excreted by the other, was observed among Lactobacillus reuteri, Bacteroides fragilis, and Escherichia coli in the utilization of xylitol. At the molecular level, we revealed that xylitol dehydrogenase (EC 1.1.1.14), xylulokinase (EC 2.7.1.17), and xylulose phosphate isomerase (EC 5.1.3.1) were key enzymes in xylitol metabolism and were present in Bacteroides and Lachnospiraceae. Therefore, they are considered keystone bacteria in xylitol digestion. Also, xylitol affected the metabolic pathway of propionate, significantly promoting the transcription of phosphate acetyltransferase (EC 2.3.1.8) in Bifidobacterium and increasing the production of propionate.

(Continued on next page)

\footnotetext{
* Correspondence: zhuxuan@zjgsu.edu.cn

†Shasha Xiang and Kun Ye contributed equally to this work.

${ }^{1}$ School of Food Science and Biotechnology, Zhejiang Gongshang University, Hangzhou 310018, China

Full list of author information is available at the end of the article
}

\section{$\triangle B M C$}

(c) The Author(s). 2021 Open Access This article is licensed under a Creative Commons Attribution 4.0 International License, which permits use, sharing, adaptation, distribution and reproduction in any medium or format, as long as you give appropriate credit to the original author(s) and the source, provide a link to the Creative Commons licence, and indicate if changes were made. The images or other third party material in this article are included in the article's Creative Commons licence, unless indicated otherwise in a credit line to the material. If material is not included in the article's Creative Commons licence and your intended use is not permitted by statutory regulation or exceeds the permitted use, you will need to obtain permission directly from the copyright holder. To view a copy of this licence, visit http://creativecommons.org/licenses/by/4.0/ The Creative Commons Public Domain Dedication waiver (http://creativecommons.org/publicdomain/zero/1.0/) applies to the data made available in this article, unless otherwise stated in a credit line to the data. 
(Continued from previous page)

Conclusions: Our results revealed that those key enzymes for xylitol digestion from different bacteria can together support the growth of micro-ecology, but they also enhanced the concentration of propionate, which lowered pH to restrict relative amounts of Escherichia and Staphylococcus. Based on the cross-feeding and competition among those bacteria, xylitol can dynamically balance proportions of the gut microbiome to promote enzymes related to xylitol metabolism and SCFAs.

Keywords: Intestinal microorganism, Xylitol, In vitro colonic simulation system (CDMN), Cross-feeding

\section{Introduction}

Xylitol is a white or transparent five-carbon polyol or sugar alcohol, which is easily dissolved in water. Xylitol is naturally distributed in fruits, vegetables, and bran but in low fractions [1-3]. In addition, a small amount of xylitol is converted in the human body [4]. Xylitol, known as an indigestible carbohydrate, is of great interest to scientists due to its prebiotic-like characteristics [5]. Several studies have investigated the relationship among the gut microbiota, xylitol, and its related metabolism. Some researches revealed that gut microbiota induced by xylitol inhibit the accumulation of lipids by synthesis of short-chain fatty acids (SCFAs) from prebiotics [6-8]. Moreover, xylitol significantly enhanced the relative amount of phylum Firmicutes and genus Prevotella, decreasing phylum Bacteroidetes and genus Barnesiella. Some researchers also found that combined supplementation of glucan and xylitol increased the concentrations of acetate and propionate and decreased the contents of branched-chain fatty acids (BCFAs) with the stability of biogenic amines by gut microbiota [9]. Xylitol also affects the metabolism of daidzein by intestinal microbial or intestinal metabolic activities. In a group of male mice supplied with $0.05 \%$ daidzein and $5 \%$ xylitol in the diet, the concentration of Bacteroidetes was decreased compared with only daidzein supplementation [10]. In addition, a single 30-g oral dose of xylitol fed to human volunteers and rodent shifted both fecal microbiome population from Gram-negative to Gram-positive bacteria [11].

Previous studies have also focused on the roles of gut microbiota involved in the metabolism of xylitol. Some researchers have reported that xylitol and sorbose can significantly promote the production of butyrate in an in vitro fermentation, which was related to an increase in the relative abundance of Anaerostipes hadrus and Anaerostipes caccae [8]. Only these two bacteria can produce butyrate derived from sorbose and xylitol among 12 typical butyrate-producing bacteria in the colon. However, further studies have found that the pure culture of Anaerostipes hadrus DSM 3319 in vitro cannot utilize xylitol [8], indicating that there exists a crossfeeding system between gut bacteria. Moreover, the growth of Streptococcus pneumoniae can be inhibited by xylitol to prevent acute otitis media in infants and young children [12,13], since xylitol cannot be metabolized by it. Xylitol decreased the level of lipopolysaccharide (LPS) on the cell membrane; thus, it inhibits the biofilm formation and growth of bacteria [14, 15]. Many studies also supported this hypothesis [16, 17], but until now, few studies discuss how xylitol alters gut bacteria. On the other hand, researches revealed that xylitol promoted the growth of beneficial bacteria, such as Bifidobacterium and Lactobacillus, in the rat colon [11, 18]. Based on its characteristics, xylitol is widely and successfully used in the biological medicines and the food industry as a sweetener [19-21]. However, the mechanism underlying the effect of xylitol on gut microbiota and its metabolism remains unknown.

A small part of dietary xylitol is absorbed by the small intestine and is metabolized by the normal metabolic pathway of the liver [22]. Over half xylitol is digested in the intestine by bacteria [23]. After xylitol is directly or indirectly converted to D-xylulose, D-xylulose-5P is further converted to fructose-6P in the pentose phosphate pathway. Subsequently, the product enters the tricarboxylic acid cycle to provide energy for cells or to be transformed into other compounds, such as ribose and succinic acid, for the usage of cells. As we know, xylitol can be converted into xylose by microorganisms such as Escherichia coli and yeast [24, 25]. Xylose can further be metabolized into pyruvic acid, which is known to be an important intermediate for the metabolism of carbohydrates into short-chain fatty acids as shown in Fig. 1. Xylitol dehydrogenase (EC 1.1.1.14), xylitol oxidase (EC 1.1.3.41), xylitol reductase (EC 1.1.1.21), and xylose isomerase (EC 5.3.1.5) are invertases in two pathways that convert D-xylitol to D-xylulose, respectively. Xylulokinase (EC 2.7.1.17) and xylulose phosphate isomerase (EC 5.1.3.1) are two important enzymes in the pentose phosphate metabolic pathway. However, few studies have reported the enzymes involved in xylitol metabolism during microbiological digestion in the colon till now.

In the previous research $[8,18,26]$, xylitol increased the concentrations of SCFAs to benefit human being. As we know, SCFAs have the ability to regulate the uptake of water and minerals, reduce the $\mathrm{pH}$ of the colon, 

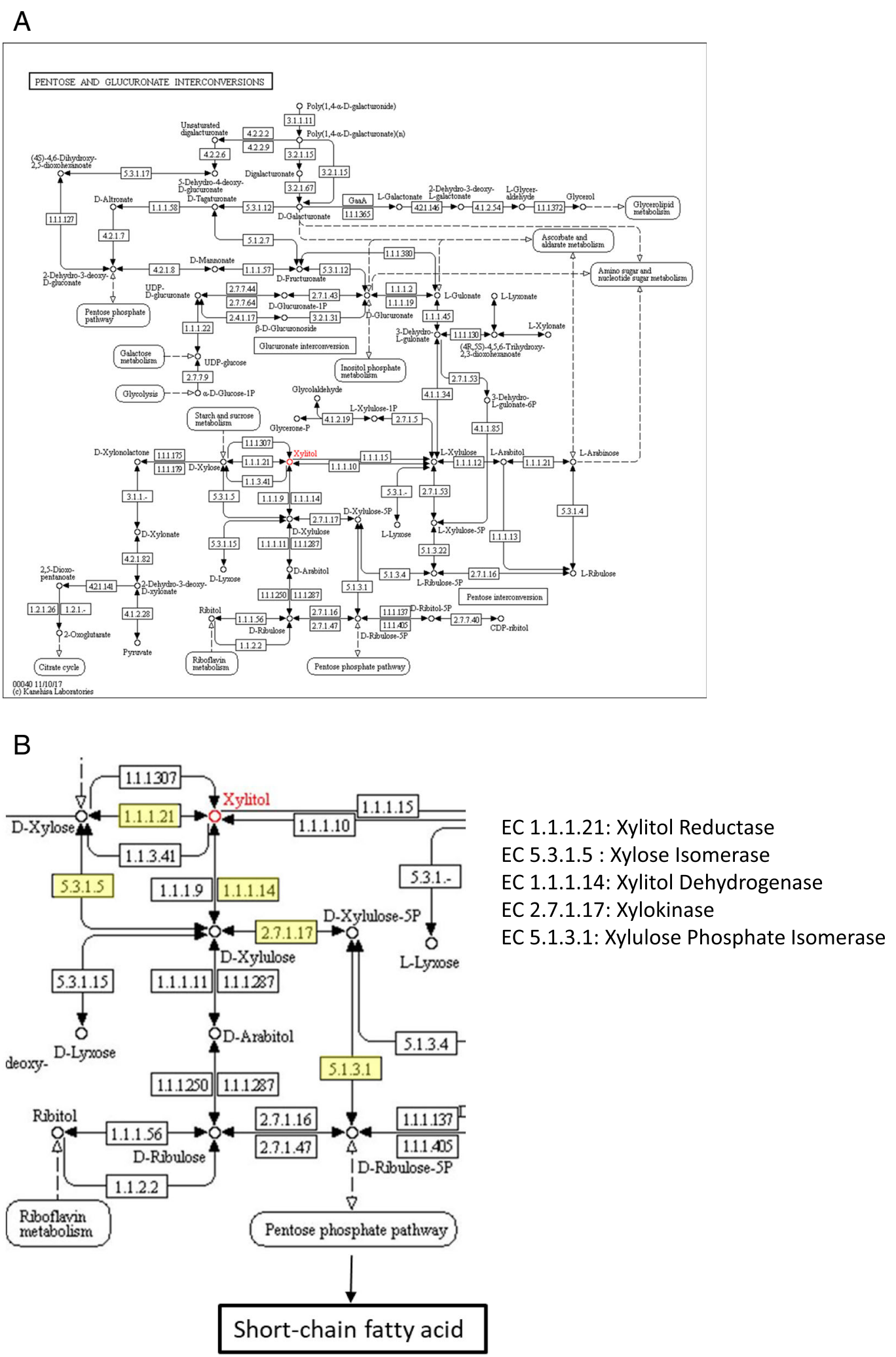

Fig. 1 Metabolic pathways of xylitol in KEGG (KEGG: Kyoto Encyclopedia of Genes and Genomes). A Overview of the pathways. B Known metabolic pathways and enzymes that xylitol is metabolized by microorganisms to produce short-chain fatty acids 
inhibit pathogens, and promote the growth of beneficial bacteria [27, 28]. Acetate is produced by most anaerobic bacteria [29] and is responsible for reducing the colonic $\mathrm{pH}$ and inhibiting the growth of pathogens. Butyrate, as a primary energy source for the intestinal epithelial cell, has many beneficial functions, such as anti-inflammation and anticancer activities, enhancing the gut barrier, inducing immune cell proliferation and differentiation, and affecting the metabolism of the energy system $[6,30]$. Propionate is mainly metabolized in the liver and inhibits cholesterol synthesis after being absorbed [30]. Propionate derived from hexose and pentose are mainly produced by the succinic pathway and can also be produced by the acrylate pathway and the propylene glycol pathway [30-33]. Some researchers demonstrated that propionate treatment in mice with a high-fat diet (HFD) reduced the body weight and systolic blood pressure, lowered fasting insulin levels, and reduced macrovesicular steatosis of the liver, hypertrophy of the liver, and inflammation of the liver induced by HFD [34]. But propionate-treated mice were more anxious in an open field and showed reduced activity of synaptogenesis and glutamate regulators in the hippocampus. However, researchers from Harvard School of Public Health also found that short-term propionate intake from food can cause high blood sugar and insulin. The long-term propionate intake caused more severe symptoms, such as obesity and insulin resistance, but propionate synthesized in the colon does not have these harmful effects [28]. Chambers et al. demonstrated that dietary fibers could prevent weight gain through promoting secretion of PYY and GLP-1 via enhancing the concentration of propionate [35]. Although propionate is beneficial to host health, the depth and comprehensiveness of studies on endogenous propionate synthesis by cross-feeding of gut flora are lacking.

Xylitol can be selectively utilized by microbiota like dietary fibers [36], polysaccharides [37], and other prebiotics $[38,39]$ to enhance beneficial metabolic pathways. However, few single species of bacteria mentioned above possess the complete genes for xylitol digestion. At present, the regulation of the interaction between xylitol, intestinal flora, and metabolic activity is still unclear. Our research combined in vivo and in vitro methodologies to study this mechanism by evaluating the effects of xylitol, its targeting point, and methods. We want to know how xylitol influences the relative abundance of beneficial bacteria and harmful bacteria. Moreover, we also want to investigate if xylitol changes metabolic activities and pathways after changing the gut microbiota composition. On the other hand, we want to understand how cross-feeding relationships are present among bacteria in the gut, which may be involved in the translation of enzymes. According to microbial metabolic pathways, especially the SCFA pathway, the transcription of related enzymes and keystone bacteria, we can understand the mechanism of the entire flora in the context of individual bacterial species. This study can be considered as a reference for xylitol further studies on the mechanisms of prebiotics and the modulation of the gut microbiota.

\section{Results}

The shift of gut microbiome in mice treated with xylitol

To determine how xylitol influences gut microbiota in the colon of mice, 8-week-old mice were provided with $0 \%, 2 \%(2.17 \mathrm{~g} / \mathrm{kg} /$ day $)$, or $5 \%(5.42 \mathrm{~g} / \mathrm{kg} /$ day) $(\mathrm{w} / \mathrm{w})$ xylitol in their chow for 3 months, the doses of which was equal to $0.18 \mathrm{~g} / \mathrm{kg} /$ day or $0.44 \mathrm{~g} / \mathrm{kg} /$ day for human being. The stools were collected monthly until sacrifice. A total of 27 samples were collected and sequenced by $16 \mathrm{~S}$ rRNA in the V4 and V5 regions. After quality control, 565,834 clean reads were obtained. In principle, an average of 47,152 $\pm 6,338$ clean reads per sample with a depth of $420 \pm 5 \mathrm{bp}$, which was an average depth of 99.8 $\pm 0.8 \%$. After a $97 \%$ similarity cluster analysis, 1521 OTUs were obtained. In the evaluation of ecological diversity, alpha diversity can reflect the abundance and diversity of species within a community. The Shannon index is used to indicate the abundance and evenness in a community. A higher Shannon index indicates higher community stability [39]. As shown in Fig. 2A, the Shannon index of the gut microbiota community of mice fed $5 \%$ xylitol in chow increased $(p<0.05)$ after 3 months. Meanwhile, the @risk v7.5.0.1 was applied to fit the data and select the optimal distribution according to Akaike's Information Criterion (AIC) standard. The probability of distribution functions (pdfs) by species under various xylitol supplementation had tiny differences (Figure S1). Moreover, the probability of distribution functions by alpha diversity under various xylitol supplementations was also plotted. The averages of functions were 2.9674, 3.2599 , and 3.3154 for $0,2 \%$, and $5 \%$ respectively (Figure S2). Most parts of these functions were overlapped, which means that no big differences were observed after xylitol consumption (Figure S2). All these results indicated that xylitol did not significantly influence the evenness and stability of the colonic community. Principal component analysis (PCA) was introduced for describing the alteration of micro-ecology after xylitol supplementation. In PC1 (33.4\%) and PC2 (30.1\%) of the twodimensional diagram, there is a difference between the xylitol group and the control after 1 month. The PCA (Fig. 2B) result indicated that xylitol caused a significant change in the gut microbiota composition.

ANOVA ( $T$ test) method was used to figure out the influence of xylitol on changing gut bacterial phylum. The relative abundance of bacteria in the colon of mice was shown in Fig. $2 \mathrm{C}$ and Figure S3. Xylitol decreased the relative abundance of Firmicutes and increased the relative abundance of Actinobacteria and Bacteroidetes 


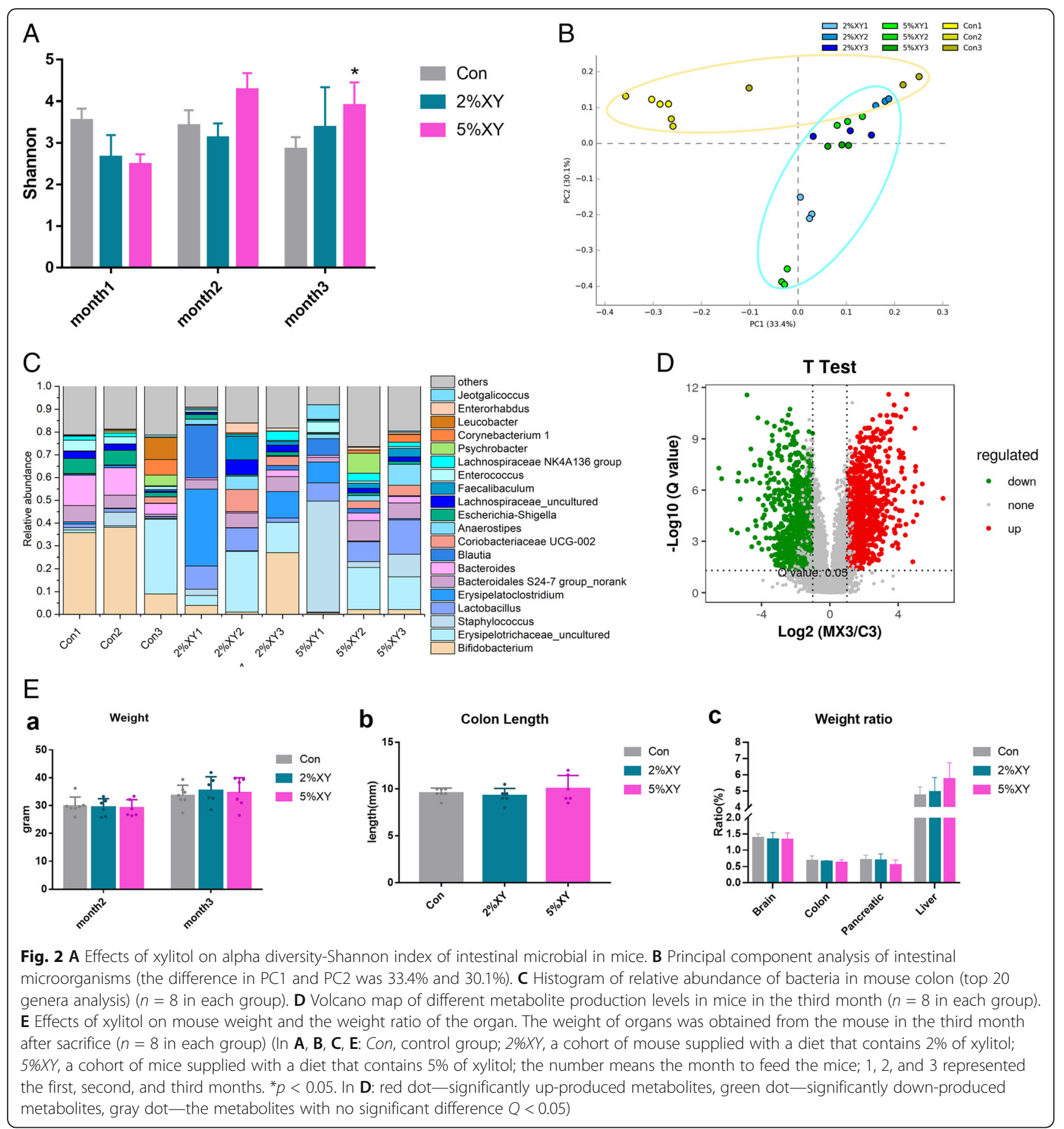

$(p<0.05)$ in the colon of mice with the increasing time. Interestingly, $2 \%$ xylitol decreased the relative abundance of Proteobacteria $(p<0.05)$ in the second month and increased it in the third month $(p<0.05)$. However, 5\% xylitol had the opposite effect. Regardless of increasing dose, Proteobacteria continued to dynamically change from $15.1 \% \pm 0.1 \%$ to $1.4 \% \pm 0.1 \%$.

The false discovery rate (FDR) method was utilized in the comparison of alternation of genera. The composition in the genus level can be found in Fig. 2C. The representative genera in each group were diverse. After 3 months, a huge decrease of Lactobacillus, Staphylococcus, Blautia, and Anaerostipes was found in the $2 \%$ xylitol supplementation group. The gut microbiota of mice treated with $5 \%$ xylitol exhibited a significant increase in the relative abundance of Bifidobacterium and Erysipelotrichaceae. Notably, a decrease in the relative abundance of Staphylococcus (from $48.6 \% \pm 3.2 \%$ to $9.8 \% \pm 1.4 \%$ ) was detected in the third month compared to that in the first month $(Q<0.05)$. The minor change also can be 
found in a decrease of the relative abundance of Enterococcus, Blautia, and Jeotgalicoccus and an increase of the relative abundance of Lactobacillus in the mice treated with $5 \%$ xylitol $(Q<0.05)$. Additionally, the relative abundance of Escherichia-Shigella, Lachnospiraceae, and Bacteroides fluctuated in both dosages of xylitol supplementation, while in the control group, we found no significant change in the composition of gut microbiota except Leucobacter over 3 months. In conclusion, 5\% $(5.42 \mathrm{~g} / \mathrm{kg} /$ day $)$ xylitol increased the relative abundance of Bifidobacterium, Lactobacillus, and Erysipelotrichaceae and decreased the relative abundance of Blautia and Staphylococcus. Lachnospiraceae remained stable. Five percent xylitol exhibited the prebiotic effect compared with $2 \%$ xylitol and control.

\section{Changes in the metabolome of mice after consuming xylitol}

To understand the influence of xylitol on metabolism, a metabolomic method and SCFA analysis were introduced to figure out the relationship between them. Compared to the control, both concentrations of xylitol promoted the production of acetate (Table S1). In the third month, only 5\% xylitol supplementation still increased the acetate concentration from $6.40 \pm 1.19$ $\mathrm{mmol} / \mathrm{g}$ to $14.49 \pm 0.11 \mathrm{mmol} / \mathrm{g}$ with a 2.3 -fold increase. This result indicated that $5 \%$ xylitol supplementation had a more significant beneficial effect. In the first 2 months, only $5 \%$ xylitol supplementation promoted the concentration of propionate $(3.23 \pm 1.06 \mathrm{mmol} / \mathrm{g}, 10.02$ $\pm 0.30 \mathrm{mmol} / \mathrm{g}$ ). Both concentrations of xylitol increased the production of propionate in the third month. Treatment with 5\% xylitol increased the propionate concentration to $10.09 \pm 0.05 \mathrm{mmol} / \mathrm{g}$ (4.6 times higher than the control). Treatment with $2 \%$ xylitol did not affect the yield of butyrate, while $5 \%$ xylitol had a significant effect on the yields of butyrate $(1.14 \pm 0.05 \mathrm{mmol} / \mathrm{g})$ and valerate $(5.61 \pm 0.03 \mathrm{mmol} / \mathrm{g})$. In summary, xylitol significantly increased the total production of SCFAs in the mouse intestine, especially $5 \%$ xylitol, which increased the output of acetate, propionate, butyrate, and valerate in the middle and later periods, while $2 \%$ xylitol supplementation had no obvious effect on butyrate.

The feces of mice in the group treated with 5\% xylitol in chow and the control mice in the third month were chosen for metabolic analyses. There were 8310 features (positive ion feature) detected, including 912 upregulated and 1152 downregulated features (Fig. 2D). The differentially produced metabolites were annotated in the KEGG pathway, and we found that xylitol significantly affected 17 metabolic pathways $(Q<0.05)$, especially the sesquiterpenoid and triterpenoid biosynthesis pathway and the level three pathway of the metabolism of terpenoids and polyketides. Among these pathways, most were involved in amino acid metabolisms, such as lysine degradation, glycine, serine, and threonine metabolism, and tyrosine metabolism analysis.

In conclusion, the in vivo experiments preliminarily evaluated the prebiotic effects of xylitol on the gut microbiota and host by promoting the growth of Bifidobacterium and Lactobacillus. In addition, xylitol increased the yield of beneficial metabolic pathways, especially propionate. Both metabolomic analysis and SCFA analysis suggested that xylitol mainly affects amino acid metabolism and promotes the concentration of propionate.

\section{Effect of xylitol consumption on physiological index}

Upon sacrifice, the brain, pancreas, colon, and liver were weighed, and the colon length was measured. To reduce the biological difference, the weight ratio of organs was calculated by organ weight divided by mouse weight. As shown in Fig. 2E, there were no significant effects $(p<$ $0.05)$ observed with $2 \%(2.17 \mathrm{~g} / \mathrm{kg} /$ day $)$ or $5 \%(5.42 \mathrm{~g} / \mathrm{kg} /$ day) xylitol. These results indicated that xylitol supplementation had no significant effect on the physiology of mice during this study.

\section{Alternation of the gut microbiome in an in vitro simulation system with xylitol supplementation}

To determine whether a cross-feeding system exists in gut microbiota from human beings during xylitol digestion, a CDMN system was used to investigate the bacterial composition and metabolism in an in vitro gut lumen and mucosa. In our study, $3 \%$ weight/volume of xylitol was utilized according to FDA instruction (Guidance for Industry Estimating the Maximum Safe Starting Dose in Initial Clinical Trials for Therapeutics in Adult Healthy Volunteers), which is equal to $0.27 \mathrm{~g} / \mathrm{kg} /$ day for a human and which is approaching to the daily recommended intake amount of xylitol. In the 1970s, the Joint FAO/ WHO Expert Committee on Food Additives (JECFA) recognized xylitol as a safe food without ADI values and the industry recommended that the daily intake should not exceed $40 \mathrm{~g}$. The CDMN was chosen to study the dynamic change of gut microbiota with the addition of xylitol, because its bacterial composition is high similar to human feces. In the gut lumen (Figure S4A), after being supplied with xylitol, the relative abundance of phyla in the gut microbiota changed substantially. We can see that in the ascending colon (AC), xylitol increased the relative abundance of Firmicutes, while it decreased the relative abundance of Synergistetes and Proteobacteria. An increase in the relative abundance of Firmicutes and a decrease in the relative abundance of Bacteroidetes and Proteobacteria were detected in the transverse colon (TC). In the descending colon (DC), the relative abundance of Firmicutes increased, while that of Proteobacteria decreased $(p<0.05)$. In summary, xylitol increased the relative abundance of Firmicutes, while it decreased 
that of Proteobacteria in the gut lumen, which was similar to the results of mice.

The FDR method was also applied in this experiment. The results of the top 20 most abundant genera in the gut microbiota of the gut lumen are shown in Fig. 3a. The predominant bacteria belong to the genus Bacteroides, Megamonas, Prevotella 9, Escherichia, and Enterococcus. The community structures of AC and DC were the most unstable after xylitol stimulation, and the relative abundance of bacteria in each genus changed greatly, which indicated that xylitol affected these two regions. Supplementation with xylitol induced a decrease in the relative abundance of Escherichia-Shigella in the ascending colon $(Q<0.05)$ and an increase in that of Megamonas $(Q<0.05)$. The microbial community structure in the transverse colon was relatively stable. Only a slight increase in the relative abundance of Blautia was detected on the 6th day. The decrease in the abundance of Escherichia-Shigella, Enterococcus, and Coprococcus was observed in the descending colon $(Q<0.05)$. Xylitol did not shift the abundance of Bacteroidetes, which was the dominated genus in all regions of the colon. Overall, xylitol decreased the proportion of Escherichia-Shigell and Enterococcus in CDMN. Surprisingly, the composition of Lactobacillus and Bifidobacterium was stable, which are in theory can easily be affected by xylitol.

There was some composition shifted in the bacteria in the gut mucosa when xylitol was added into CDMN (Figure S4B). In the ascending colon, the relative abundance of Actinobacteria and Firmicutes increased, while that of Proteobacteria decreased. No significant change was detected in the abundance of Bacteroidetes. In the
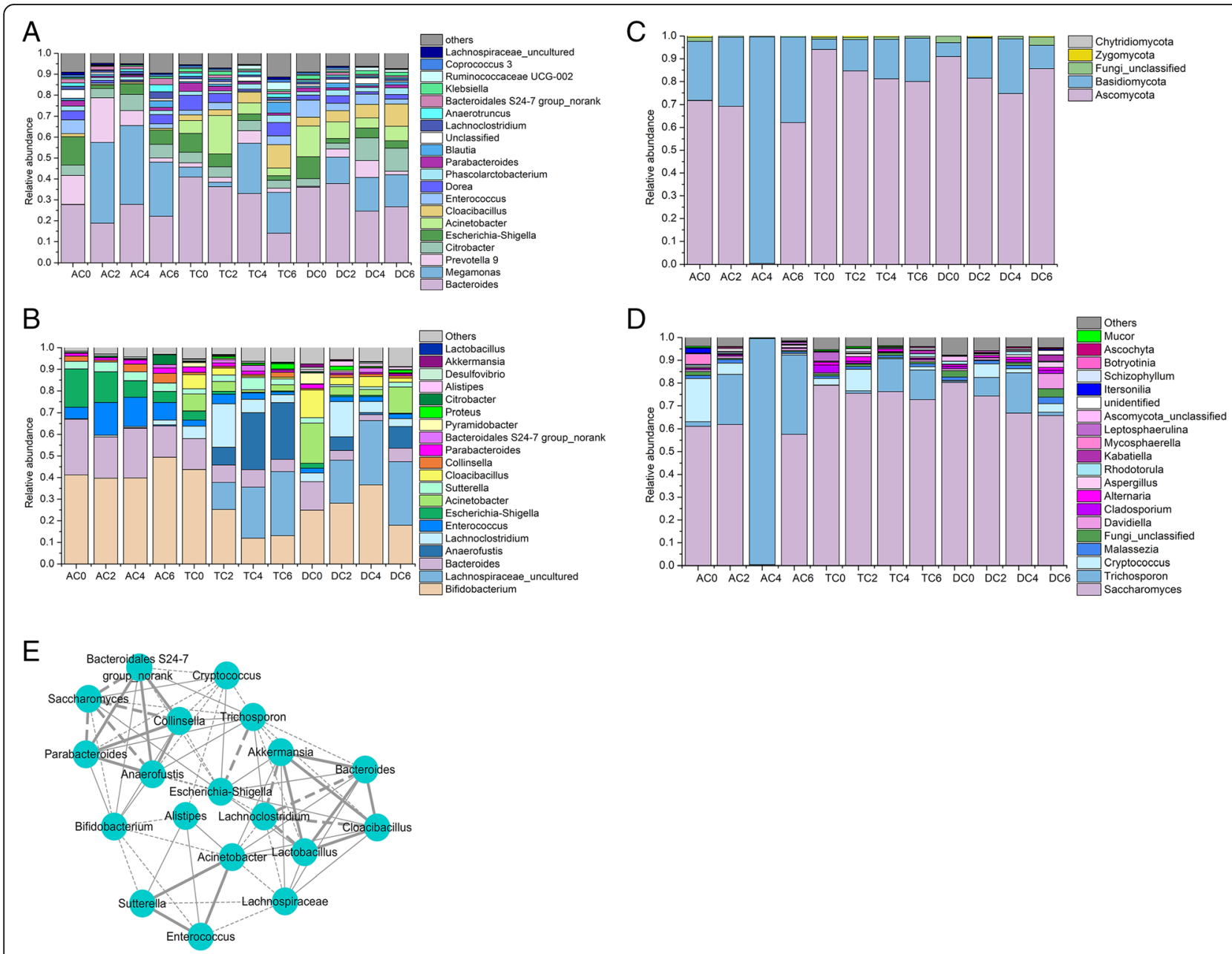

Fig. 3 A Histogram of relative abundance of bacteria in different parts of in vitro colon (top 20 genera of bacteria in the lumen). B Histogram of relative abundance of bacteria in different parts of in vitro colon (top 20 genera of bacteria in the mucosa). $\mathbf{C}$ Histogram of relative abundance of fungi in different regions of the colon (phylum). $\mathbf{D}$ Histogram of relative abundance of fungi in different regions of the colon (top 20 genera of fungi in the lumen). E Spearman correlation analysis of microorganisms (solid line-positive correlation, dotted line - negative correlation, thick line-significant correlation $p<0.01$. AC, TC, and DC presented the ascending colon, transverse colon, and descending colon; the number means the days for adding xylitol) 
transverse colon, the fraction of Firmicutes increased, while that of Actinobacteria and Proteobacteria decreased, with no significant difference in that of Bacteroidetes. An increase in the relative abundance of Actinobacteria and Firmicutes and a decrease in the relative abundance of Bacteroidetes and Proteobacteria were detected in the descending colon. The above significant value was $p<0.05$. The top 20 genera in the gut mucosa are shown in Fig. 3b and the FDR method was also applied. Bifidobacterium, Lachnospiraceae, Bacteroides, Anaerofustis, and Enterococcus were the dominant genera in the mucosal layer. The most unstable community structure in the mucosa was found in the transverse colon and descending colon, indicating that xylitol had a high influence on microbiome in the mucosa of the large intestine. Xylitol increased the fraction of Parabacteroides, Bacteroidales S24-7 group_norank, Lachnoclostridium, Citrobacter, and Anaerofustis in the mucosa of the ascending colon. While the relative abundance of Escherichia-Shigella and Desulfovibrio decreased in the same region on the last day. Xylitol had more effects on the transverse colon and descending colon. After 6-day supplementation, a significant decrease in the relative abundance of Bifidobacterium, Escherichia-Shigella, Enterococcus, Desulfovibrio, and Alistipes was detected and a huge increase can be observed in the relative abundance of Proteus, Citrobacter, Bacteroidales S24-7 group_norank, Anaerofustis, and Lachnospiraceae. In the descending colon, the dramatic change of microbial community can be found on the 4th day. The relative abundance of Bifidobacterium, Lachnoclostridium, Lachnospiraceae, and Anaerofustis were enriched, while the relative abundance of Bacteroides and Escherichia-Shigella were decreased. There was no significant difference in the change of the relative abundance of Lactobacillus in the three regions of the colon. In general, xylitol increased the fraction of Lachnospiraceae and decreased that of Escherichia-Shigella in each intestinal region.

As we know fungi were usually involved in the metabolism of xylitol, the ITS method was used to investigate the relationship between xylitol and fungi. Ascomycota and Basidiomycota accounted for $95 \%$ of the fungi in the human gut. More than $60 \%$ of fungi were members of the Ascomycota phylum (Fig. 3c) and represented by Saccharomyces. Xylitol mainly altered the fungi composition in AC, especially on the 6th day. The relative abundance of Saccharomyces, Malassezia, Cryptococcus, Cladosporium, and Botryotinia was higher on the 6th day than that on the 4th day, while a huge decrease of the relative abundance of Trichosporon was observed, decreasing from 99.12 to $34.67 \%(Q<0.05)$. Xylitol also induced the composition alteration in TC. An increase in the relative abundance of Cryptococcus, Wallemia, and Eutypa and a decrease in the fraction of Mucor and
Microascus were detected on the 6th day compared to that on the 4th day $(Q<0.05)$. No significant change can be found in TC. In conclusion, xylitol decreased the relative abundance of Saccharomyces. In contrast, xylitol increased the fraction of Trichosporon (Fig. 3d). The human commensal Trichosporon spp. can adapt to the host microenvironment and its immune system [40].

After analysis of the Spearman correlation among microbiota, the relationship and interaction among gut microbiota were determined, as shown in Fig. 3e. There were three significant interaction networks. Saccharomyces negatively modulated Bacteroidales S24-7 group_norank, Collinsella, Parabacteroides, and Anaerofustis in the network. Lachnoclostridium negatively modulated Akkermansia, Lactobacillus, Bacteroides, and Cloacibacillus. There was a positive network among Acinetobacter, Sutterella, and Enterococcus. Although Bifidobacterium did not join any of the three significant interaction networks, it had a close relationship among them. Bifidobacterium was associated with three bacterial interaction networks of Saccharomyces and Acinetobacter. In addition, it was also associated with the network of Lachnoclostridium by connection with Anaerofustis. Bifidobacterium negatively correlated with the network of Lachnoclostridium and Acinetobacter. Escherichia-Shigella had a positive correlation with Lactobacillus and Bacteroides. Regardless of the pattern by which Escherichia-Shigella interacted with other bacteria, Bifidobacterium always inhibited it. In addition, Bifidobacterium was negatively correlated with the SCFA-producing bacteria Lachnospiraceae and Anaerofustis. Those results indicated that several genera of bacteria or fungi were involved in xylitol metabolism by a cross-feeding system. Regarding Spearman analysis, the whole network of microbiota was divided into several parts, in which genera EscherichiaShigella, Lactobacillus, Bifidobacterium, and Bacteroides dominated key positions and had close interactions with many bacteria in the community.

\section{Changes of metabolome induced by xylitol in an in vitro simulation}

In order to investigate the alternation of the metabolome, an electronic sensor was introduced to examine the alterations of gases from CDMN during the experiment. Ammonia, hydrogen sulfide, hydrogen, and methane are the primary components of intestinal gases (Figure S5), which were converted from different nutrients in the intestinal medium. The gas analysis can preliminarily reflect the metabolism of the gut microbiota. There was no significant difference in the gas composition in the ascending colon compared with the baseline level of gas. The sulfide (SE2), hydrogen (SE3), and methane (SE14) in the transverse colon decreased. Less microbial ammonia (SE1) was produced in the descending colon. 
In addition, the SCFA analysis was also applied to investigate the relationship between xylitol and microbiota. The concentration of SCFAs in the mucosa was twice higher than that in the lumen (Fig. 4A). Xylitol significantly increased the yield of SCFAs both in the lumen and mucosa. However, the types and distribution of SCFAs varied. The concentrations of propionate in the colon and butyrate in the mucosal layer were increased by xylitol. On the 6th day, compared to the baseline level, the propionate level increased by 2.1 folds from $13.6 \pm 1.5$ $\mathrm{mmol} / \mathrm{L}$ to $28.4 \pm 2.1 \mathrm{mmol} / \mathrm{L}$ in the lumen of the ascending colon. In the mucosa of the transverse colon, the yield of butyrate increased from $19.5 \pm 3.4 \mathrm{mmol} / \mathrm{L}$ to $68.5 \pm$ $4.5 \mathrm{mmol} / \mathrm{L}$. The concentration of propionate in the lumen increased to $43.1 \pm 2.3 \mathrm{mmol} / \mathrm{L}$, and the concentration of butyrate in the mucosa of the transverse colon increased to $113.0 \pm 5.6 \mathrm{mmol} / \mathrm{L}$ in the treated group compared to the data at $T=0$ (time). In conclusion, the

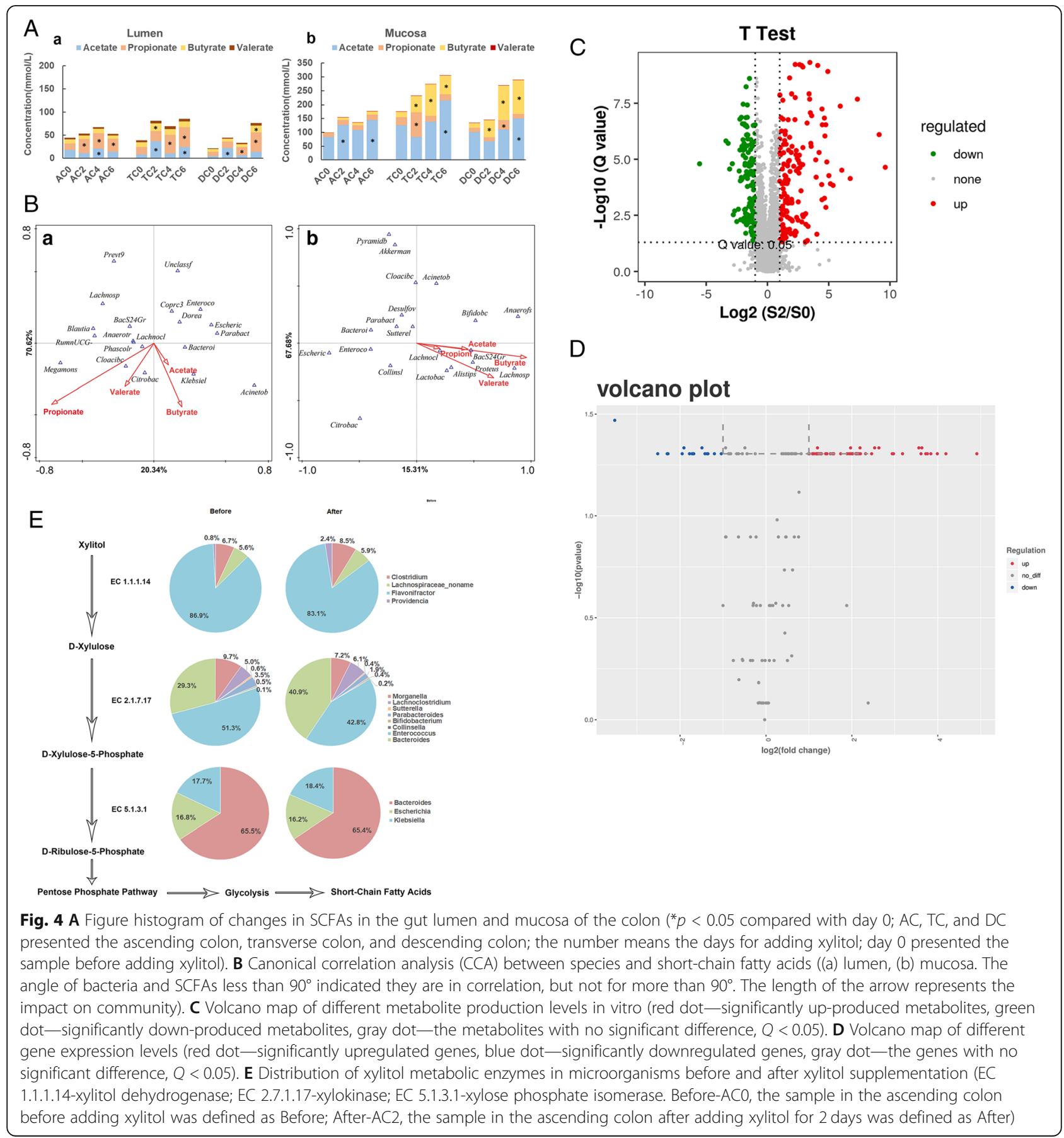


in-site propionate was synthesized from xylitol by gut microbiota. Therefore, it is mainly produced from the microbiota in the mucosa for the rapid utilization.

Canonical correlation analysis (CCA) is a statistical analysis that comprehensively describes the correlation between two variables. In this study, the correlation between the relative abundance of the gut microbiota and the concentration of SCFAs was used for CCA, which can attribute the production of SCFAs to species. As shown in Fig. 4B (a), propionate and valerate were distributed in the third quadrant, while acetate and butyrate were distributed in the fourth quadrant. Most microbiota was in the first, second, and third quadrants. The angle between propionate and almost all microorganisms in the second and third quadrants was sharp. This result indicated that many bacteria, such as Lachnospiraceae, Megamonas, Lachnoclostridium, and Cloacibacillus, were involved in the production of propionate. However, only limited bacteria contributed to the production of butyrate. In the mucosal layer (Fig. 4B (b)), SCFAs were distributed in the fourth quadrant, and the butyrate production in the mucosa was the highest, which is mainly related to the microorganisms in the first and second quadrants, including Bifidobacterium, Anaerofustis, Sutterella, Bacteroidales S24-7 group norank, and Citrobacter in addition to the reduced relative abundance of Escherichia-Shigella. Interestingly, in either the lumen or mucosa, Escherichia-Shigella and Enterococcus were not associated with the production of SCFAs, which was in accordance with the results of mice

\section{Metabolomic profile of samples in an in vitro simulation}

From the results of the gut microbiota composition, the community in the ascending colon shifted substantially, especially on the beginning day and 2nd day. Therefore, the metabolomic profiles of these samples were selected for analysis. There were 8310 features detected, including 161 upregulated and 145 downregulated features (Fig. 4C). In total, 17 pathways showed significant differences with the addition of xylitol $(Q<0.05)$. Most of them were catalogized into the secondary pathway of amino acid metabolism and metabolism of other amino acids (5 tertiary pathways) and carbohydrate metabolism (7 tertiary pathways). Although there was no significant difference observed in the propionate pathway $(Q=0.0596)$, we found that metabolites such as methylglyoxal, 2-methyl-cis-aconitate, and 2-methyl-trans-aconitate were enriched (Figure S6). For pentose and glucuronate interconversion $(Q=$ $0.167)$, the production of downstream products of xylitol conversion, such as D-arabitol and ribitol, were enhanced (Figure S7). The results of xylitol digestion pathway analysis from the metabolome coincided with those of the metatranscriptome.

\section{Effect of xylitol on the metatranscriptome of colonic microorganisms}

In consistence with metabolomic analysis, the same samples were selected and collected for metatranscriptome analysis. The effect of xylitol on the transcriptome of colonic microorganisms is shown in Fig. 4. The differentially expressed genes after transcriptomic sequencing were annotated by GO. The distribution of differentially expressed genes was presented in a volcano map in Fig. 4D. In total, 29,224 types of genes were detected and 12,926 types of genes showed significant changes in this study, including 8930 upregulated genes (red) and 3996 downregulated genes (blue). After gene sequencing, assembly, and annotation, the distribution of enzymes based on xylitol digestion in the microbiota is shown in Fig. 4E. The quantity of enzyme related with xylitol were all increased after xylitol supplementation (Fig. 5A). Xylitol dehydrogenase (EC 1.1.1.14), xylulokinase (EC 2.7.1.17), and xylose phosphate isomerase (EC 5.1.3.1) were the basic digestive enzymes of xylitol, and the microorganisms that can express these enzymes are considered the basis of xylitol digestion in bacteria. The distribution of xylulokinase (EC 2.7.1.17) in intestinal microorganisms was the most abundant, followed by xylitol dehydrogenase (EC 1.1.1.14) and xylose phosphate isomerase (EC 5.1.3.1). However, not all enzymes exist in a single bacterium. For example, xylitol dehydrogenase (EC 1.1.1.14) was mainly distributed in Flavonifractor, Clostridium, Providencia, and Lachnospiraceae. Among them, only Lachnoclostridium had xylulokinase (EC 2.7.1.17). In the pie chart of the distribution of xylulose phosphate isomerase (EC 5.1.3.1), no bacteria were found in the family of Lachnospiraceae. We suggested that the microorganism with the enzyme xylitol dehydrogenase (EC 1.1.1.14) transformed xylitol into xylose and transferred xylose to the microorganism containing xylulokinase (EC 2.7.1.17). The metabolites were further transferred to the bacteria with xylose phosphate isomerase (EC 5.1.3.1) for gradual utilization. This result further proved that there was a cross-feeding system in the microbiota related to xylitol digestion. After the ingestion of xylitol, the distribution of enzymes in microbiota also changed. The proportion of xylitol dehydrogenase (EC 1.1.1.14) in Flavonifractor decreased from 87 to $83 \%$. In addition, the fraction of Lachnospiraceae, Clostridium, and Providencia increased. The proportion of xylulokinase (EC 2.7.1.17) in Bacteroides and Lachnoclostridium increased from $29 \%$ and $5 \%$ to $41 \%$ and $6 \%$, respectively. The change in the distribution of xylulose phosphate isomerase (EC 5.1.3.1) was limited, with only a $1 \%$ difference between Escherichia and Bacteroides. Xylitol dehydrogenase (EC 1.1.1.14) and xylulokinase (EC 2.7.1.17) were likely two key enzymes in the microbial digestion of xylitol, while Lachnospiraceae bacteria also had the ability to translate these two enzymes, as key enzymes for 


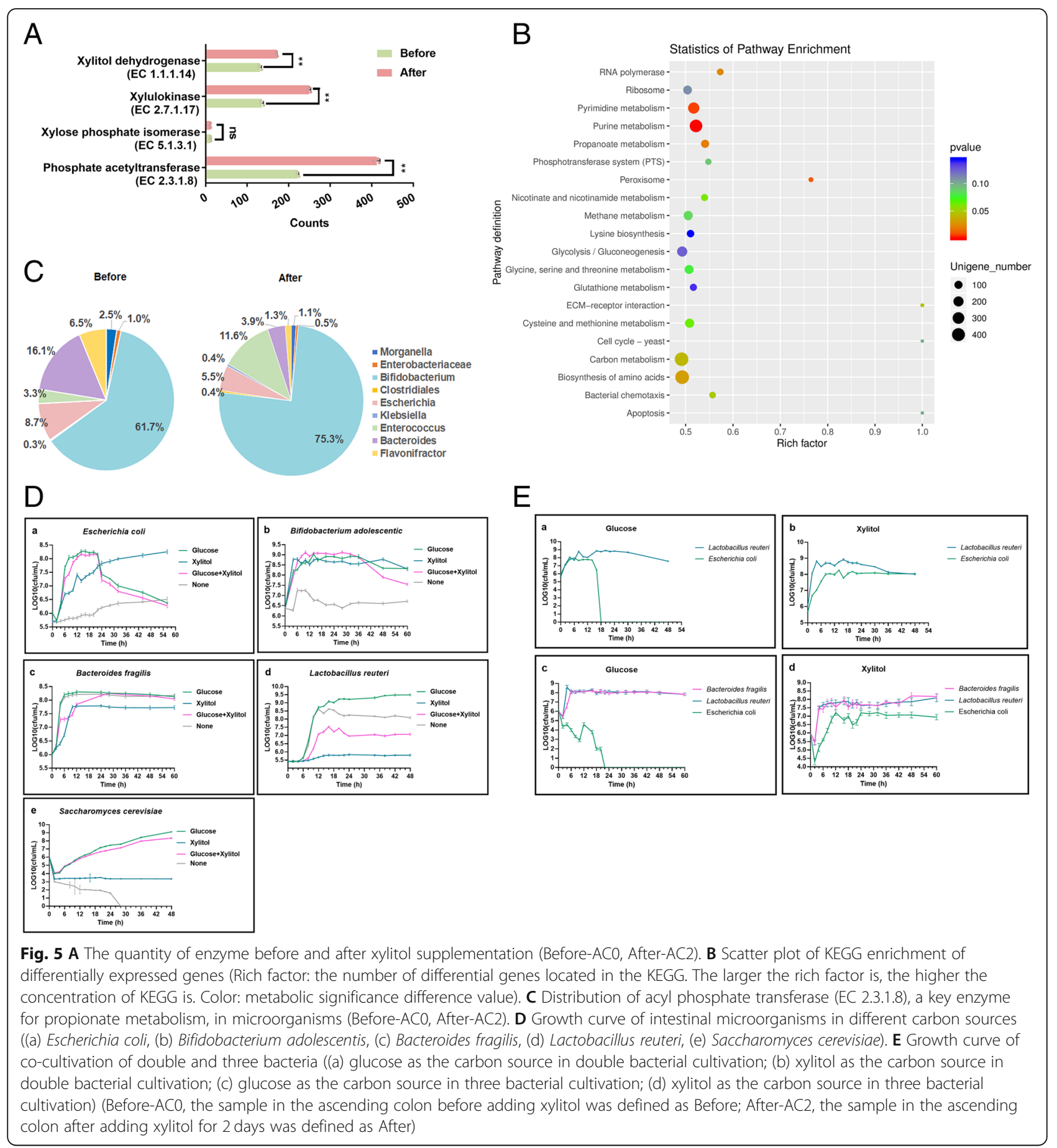

bacterial xylitol digestion. Bacteroides was also important in xylitol digestion because it had the ability to translate xylulokinase (EC 2.7.1.17) and xylulose phosphate isomerase (EC 5.1.3.1).

Enrichment analysis of the functions of differentially expressed genes can reveal the function of microorganisms. The pathway enrichment analysis was performed based on the KEGG database. The significantly enriched pathways are shown in Fig. 5B. We found 9 pathways with significant differences $(p<0.05)$ in propionate, purine, and carbohydrate metabolism. Amino acid synthesis was the most abundant and significantly changed pathway. The results showed that xylitol digestion not only changed the composition of the gut microbiota, but also affected the metabolic pathways of other nutrients.

Xylitol promoted the production of propionate, upregulating 66 genes and downregulating 31 genes, including the upregulation of the transcription of a limiting 
enzyme associated with propionate synthesis in the succinic acid pathway (Figure S8). There were 2 enhanced pathways from propionyl-CoA to propionate. One pathway required 2 steps for phosphorylation, while the other required one step for direct conversion. Phosphate acetyltransferase (EC 2.3.1.8) was the limiting enzyme of propionate metabolism, which was mainly distributed in Bifidobacterium, Bacteroides, Escherichia, and Enterococcus (Fig. 5C). The quantity of this enzyme was increased (Fig. 5A) in this study.

Xylitol induced a shift in the enzyme distribution in microorganisms. The proportions of enzymes in Bifidobacterium and Enterococcus increased from 62\% and $3 \%$ to $75 \%$ and $8 \%$, respectively, with decreasing in the proportions of enzymes in other bacteria, especially Escherichia and Bacteroides, which was similar to the relative abundance of these bacteria in the gut. Therefore, Bifidobacterium and Enterococcus were potential key bacteria that promoted the production of propionate. However, from the results of CCA, we knew that Enterococcus had no relationship with SCFA production. In conclusion, Bifidobacterium was vital for propionate production. Propionate CoAtransferase (EC 2.8.3.1), which functioned in the onestep pathway, was present only in Flavonifractor. The transcriptomes were upregulated after the ingestion of xylitol in the experiments, indicating that Flavonifractor mainly converted propionyl-CoA to propionate with the help of propionate CoA-transferase. Our results revealed that those key enzymes for xylitol digestion from different bacteria can together support the growth of micro-ecology, but they also enhanced the concentration of propionate, which lowered $\mathrm{pH}$ to restrict relative amounts of Bacteroides and Escherichia. Based on the cross-feeding and competition between those bacteria, xylitol can dynamically balance proportions of the gut microbiome to promote relative abundances of beneficial bacteria and SCFAs.

\section{Xylitol as a carbon source for single-strain cultivated and co-cultivated bacteria}

Emerging evidence indicated the saccharides or prebiotic can be used by colonic bacteria solely or by crossfeeding among two or several bacteria [41]. To confirm whether xylitol can be used by single bacteria or not, the single-strain cultivation and co-cultivation methods were introduced. Gut bacteria were selected according to the network made by Spearman analysis, which showed the importance of bacteria in the gut microbiota for xylitol digestion. The basic nitrogen medium was prepared with or without the sole carbon sources of glucose and xylitol or the combination of glucose and xylitol for the cultivation of Escherichia coli, Bifidobacterium adolescentis, Bacteroides fragilis, Lactobacillus reuteri, and
Saccharomyces cerevisiae. As we know, Lactobacillus reuteri and Saccharomyces cerevisiae did not contain enzymes related to xylitol metabolisms. The results of xylitol consumption are shown in Fig. 5D. There was no difference in the growth of $E$. coli in any of the four media at the early stage. However, the number of $E$. coli in the medium with glucose as the carbon source started to decline at $20 \mathrm{~h}$, but this trend was not observed for the other two media. This result illustrated that E. coli had the capacity to use carbon and nitrogen sources. In addition, because $E$. coli was also able to use xylitol for growth to stabilize its biomass, no rapid decline was observed in the medium with a complex carbon source. Compared with the negative control, B. adolescentis grew on medium with only xylitol and showed no significant difference in growth on the other media (Fig. 5D (b)). These results showed that xylitol can be used by $B$. adolescentis as a carbon source. Although B. fragilis survived on the xylitol medium, it did not grow as well as it did on the media with glucose or without a carbon source. It means that xylitol inhibited the growth of $B$. fragilis (Fig. 5D (c)). L. reuteri had limited growth on the xylitol medium. Beyond our expectation, L. reuteri with a combination supplementation of glucose and xylitol showed a low growth rate in the first $30 \mathrm{~h}$, compared with glucose supplementation. Therefore, xylitol cannot be directly used by $L$. reuteri and further influenced the carbon metabolism of L. reuteri (Fig. 5D (d)). Although it rapidly declined on the medium lacking a carbon source, $S$. cerevisiae grew weakly in xylitol medium, but far less than it did on glucose as a carbon source, which showed that $S$. cerevisiae uses xylitol weakly (Fig. 5D (e)).

The growth of bacteria in co-cultivation medium is shown in Fig. 5E. L. reuteri and E. coli were grown in a medium containing glucose. However, the growth of $E$. coli was inhibited in the later period because of the acidification of the medium by $L$. reuteri (Fig. 5E (a)). Interestingly, on xylitol medium, we found that $L$. reuteri, which could not use xylitol in the single-strain cultivation, grew well and that the number of $L$. reuteri was much higher than the number of $E$. coli (Fig. 5E (b)). This result indicated that E. coli can support $L$. reuteri to use xylitol without much acidification. The growth situation in the co-cultivation of three organisms was similar to that in the co-culture of two species. On glucose medium, $L$. reuteri also inhibited the growth of $E$. coli by acid production but had no effect on B. fragilis. The growth rate of $E$. coli was always lower than that of $L$. reuteri and $B$. fragilis. This indicates that $L$. reuteri and $B$. fragilis were dominant bacteria, but xylitol had no effect on the community structure (Fig. 5E). In conclusion, a cross-feeding system involved into the utilization of xylitol by gut microbiota. 


\section{Discussion}

Understanding how xylitol works as a prebiotic for human beings is critical in xylitol application. In this study, we evaluated the mechanism by which xylitol influenced the gut microbiota, metabolic activity, and beneficial metabolism markers in vivo and in vitro. Collectively, according to the microbiota, metabolome, and metatranscriptome results, we revealed the mechanism of the effect of xylitol on propionate synthesis at the molecular level.

As a result, probability distribution functions (pdfs) of relative species data (RSD) indicated that no great difference between various concentrations of xylitol supplementations was found. Meanwhile, the pdfs of alpha diversity also demonstrated that xylitol had little influence on the diversity of the microbiome. Both results exhibited a no-linear effect of xylitol on the microbiome, which was similar with the results of Lijie [42]. Lijie et al. stated that those profiles of pdfs can be used to identify the situation of the microbiome. In this case, we found that xylitol had no influence on the composition of micro-ecology and further little influenced on the physiology of mice, which was also supported by our experiment. As a result, some bacteria should play a key role in xylitol metabolism. In the mouse experiments, we found that $5 \%(0.44 \mathrm{~g} / \mathrm{kg} /$ day for a human being) xylitol in chow promoted the evenness and maintained the stability of the micro-ecology in the gut. However, a difference was detected in the $5 \%$ xylitol in the chow treatment group. The consumption of $5 \%$ xylitol increased the relative abundance of Bifidobacterium and Lactobacillus, maintained the concentrations of Bacteroides and Lachnospiraceae, and decreased the relative abundance of Escherichia and Staphylococcus (Fig. 2D). However, a high relative amount of Staphylococcus was found in the group of $0.44 \mathrm{~g} / \mathrm{kg} /$ day (for human being) xylitol in chow in the first month. The genus Staphylococcus contains not only pathogen Staphylococcus aureus, but also symbiotic Staphylococcus carnosus, which can be used as a start culture for sausage fermentation [43]. Until sacrifice, we did not find significant differences in the physiology of mice fed either $2 \%(0.18 \mathrm{~g} / \mathrm{kg} /$ day for a human being) or $5 \%$ xylitol in their chow, indicating that a novel stability of gut-ecology was formed through propionate synthesis after a long-term xylitol supplementation. Although microbiota in mice are varied with that in human beings, their advantages are extensive and the breadth and depth of research on mouse gastroenterology, immunology, and genetics largely exceed any other model $[44,45]$. A reasonable diet, suitable species, and stable environment factors can enhance the similarity and repeatability of the human gut microbiota shifts in mice. Mouse experiments of xylitol were used to investigate potential xylitol mechanisms and their interplay between host, microbial, and environmental factors. Considering the in vivo results and calculations according to FDA introduction (Guidance for Industry Estimating the Maximum Safe Starting Dose in Initial Clinical Trials for Therapeutics in Adult Healthy Volunteers $)$, 3\% xylitol $(0.27 \mathrm{~g} / \mathrm{kg} /$ day for a human being) was applied in an in vitro experiment to investigate microbial digestion of xylitol in microbiota from a human being colonic simulation. In general, xylitol maintained the fraction of Lactobacillus and Bifidobacterium, while it decreased that of Escherichia-Shigella in each intestinal region, and the relative amount of Lachnospiraceae was increased in the mucosal layer (Fig. 3a, b). Among them, Bifidobacterium and Lactobacillus are the most wellknown beneficial bacteria in the gut. The genus Bifidobacterium contains 80 species, which are distributed across different niches and an important bacterium for propionate synthesis, including the human gastrointestinal tract. Bifidobacterium rapidly becomes the dominant bacteria in the infant's gut after birth because of milk-derived oligosaccharides (human milk oligosaccharides) that simulate the proliferation of Bifidobacterium [46]. Lactobacillus species, such as L. reuteri, L. plantarum, and L. rhamnosus, are widely used as probiotics to prevent gut dysbiosis [47], although they do not produce propionate. But, the selective stimulation of Bifidobacterium and Lactobacillus by prebiotics has been widely shown, and Bifidobacterium and Lactobacillus are important biomarkers for healthy gut micro-ecosystems $[48,49]$. Those bacteria were also reported to support the growth of each other [50]. The selective enhancement of these two genera of beneficial bacteria is considered to be the reason why prebiotic therapies could be beneficial for children who have developed attention deficit hyperactivity disorder [51]. Lachnospiraceae, which was changed significantly in our study, is a family of Clostridia, some species of which have the capacity to produce butyrate in the gut. Conor et al. analyzed the genome of 30 sequenced Lachnospiraceae and found that only 12 of them had the gene annotated from at least one enzyme, butyrate kinase (from butanol-p) or BCoAT (from butanol-CoA), allowing the production of butyrate [52]. Some researchers also reported that Lachnospiraceae involve in hydrolyzing glucosides and degrading pectin and cellulose [53]. In our study, all those bacteria, induced by xylitol, were related to SCFAs, especially propionate synthesis.

The concentrations of SCFAs in the gut lumen and mucosa were quantified in vitro. We found that the shift in the SCFAs in CDMN coincided with that in mice. The SCFAs in the mouse feces primarily reflected the SCFAs in the colonic lumen. Acetate and butyrate were mainly distributed in the mucosa (Fig. 3e), indicating that they were responsible for host health by lowering the $\mathrm{pH}$ of the mucosa to inhibit the adhesion and 
proliferation of pathogens. The enrichment of SCFAs, especially propionate and butyrate, is in agreement with the research of Tadashi [8]. Additionally, propionate was used to balance the micro-ecosystem in the lumen. The results of the bacterial composition in the lumen and mucosa supported this phenomenon.

Several studies have also clarified the prebiotic effects of xylitol from various perspectives $[7,8,10,11,26]$. In our study, xylitol promoted some bacteria or their corresponding enzymes capable of digesting xylitol, inhibited the growth of Escherichia, increased the content of SCFAs, and enhanced the adhesion of Bifidobacterium. Tadashi et al. revealed that xylitol promoted the production of propionate and butyrate using human fecal culture and demonstrated that Anaerostipes hadrus and Anaerostipes caccae augmented butyrate production from xylitol [8]. Tamura et al. suggested that dietary xylitol can affect bone health by enhancing isoflavonoid production via altering the metabolic activity of intestinal microbiota and gut environment [10]. The combined application of Lactobacillus and xylitol has been shown to prevent Clostridium difficile infection by translocating the colonic mucosa morphology and shifting the gut microbiota [54]. The human study in 1985 provides solid evidence to confirm the prebiotic effects of xylitol on humans [11]. But all of those studies did not tell us how xylitol is utilized and metabolized by microbiota.

Based on results from metatranscriptomic and metabolomic analysis, it could be concluded that the digestion of xylitol was performed by the co-operation of several gut bacteria. Xylitol dehydrogenase (EC 1.1.1.14), xylulokinase (EC 2.7.1.17), and xylulose phosphate isomerase (EC 5.1.3.1) were key enzymes in xylitol metabolism and were present in Bacteroides and Lachnospiraceae. Phosphate acetyltransferase (EC2.3.1.8), which is a crucial enzyme for propionate synthesis, was mainly distributed in Bifidobacterium and Escherichia in the presence of xylitol. They all supported the energy metabolism and growth of micro-ecology, but on the other hand, they enhanced the concentration of propionate, which can lower $\mathrm{pH}$ to restrict the growth of Bacteroides and Escherichia. It indicated that xylitol can dynamically balance the relative amount of the gut microbiome. This kind of interaction of microbiota that coexists in nature has a profound role in human health. Three kinds of inter-species interactions are defined as beneficial, neutral, or harmful effects on gut-ecology. Regarding recent studies, a novel graph emerged, which emphasizes convoluted networks and inter-dependencies of microbiomes. Those microbiomes are not limited to exchanges of electron donors for growth, but include the exchanges of vitamin, amino acids, siderophores, and other cofactors [50]. Monosaccharides, such as xylitol, xylulose, and 5-P-xylulose, are absorbed by microorganisms through a reversible transport way [55]. High concentrations of xylulose and 5-Pxylulose can be transported outside the cell as a substrate for other microorganisms. Studies have shown that xylitol dehydrogenase (EC 1.1.1.14) is a dehydrogenase, which uses NADP $+(\mathrm{H})$ as a coenzyme $[56,57]$. Catalytic dehydrogenation can produce a large amount of NADPH and $\mathrm{H}+[58]$, which can be used for the synthesis of nucleic acids, fatty acids, and ATP to maintain the normal biological activities of microorganisms. Thus, interacting partners of microorganisms in the cross-feeding system use xylitol not only to supply electron donor exchange but also to provide suitable carbon sources and nutrition. In order to further understand the function of xylitol, singlestrain bacterial cultivation and co-cultivation experiments were conducted. In the experiments of single-strain bacterial cultivation and co-cultivation, the usage of xylitol by L. reuteri and E. coli were different (Fig. 4C, D). The cross-feeding, a relationship in which one organism consumes metabolites excreted by another, is a ubiquitous feature of natural microbial communities and can be a key factor in creating complex communities and promoting diversity in environments [59]. The co-cultivation experiments revealed a cross-feeding relationship among $L$. reuteri, E. coli, and B. fragilis. In addition, from the molecular analysis, we found that xylitol dehydrogenase (EC 1.1.1.14) was a core enzyme that converts xylitol. Interestingly, xylitol dehydrogenase (EC 1.1.1.14) is not distributed in $E$. coli, although E. coli is able to use xylitol [58]. In our study, the survival bacteria depend to some extent on the whole microbiome to guarantee carbon flow and exchange its by-products. A study using a gnotobiotic mouse model also demonstrated that extracellular digestion of inulin increased the growth rate of Bacteroides ovatus, the byproducts of which from inulin catabolism were utilized by primary fermenters such as Faecalibacterium prausnitzii and Bacteroides vulgatus [60]. The sequential action of a serial of gut microbiota involving fermentation pathways generates the metabolic input for a diverse set of gut microbiota. In addition, electron donor exchanges also contributed to explaining the dynamic interactions $[11,54,58]$.

In nature, xylitol, as an intermediate product converted from D-xylose to xylulose, is found in fruits, vegetables, mushrooms, seaweed, and yeast. Different species of yeast are able to ferment xylose to xylitol or ethanol at low oxygen levels with redox imbalance. In Debaryomyces hansenii, the low ratio of NAD/NADH in the oxidation step enables the production of xylitol. Pichia stipites, with xylose reductase, can generate NAD+ to convert xylitol to ethanol [60]. But in our study, the low concentrations of fungi and yeast did not make a big contribution to xylitol digestion.

On the other hand, due to the spatial difference for microbiota between lumen and mucosa, we found that microbiota 


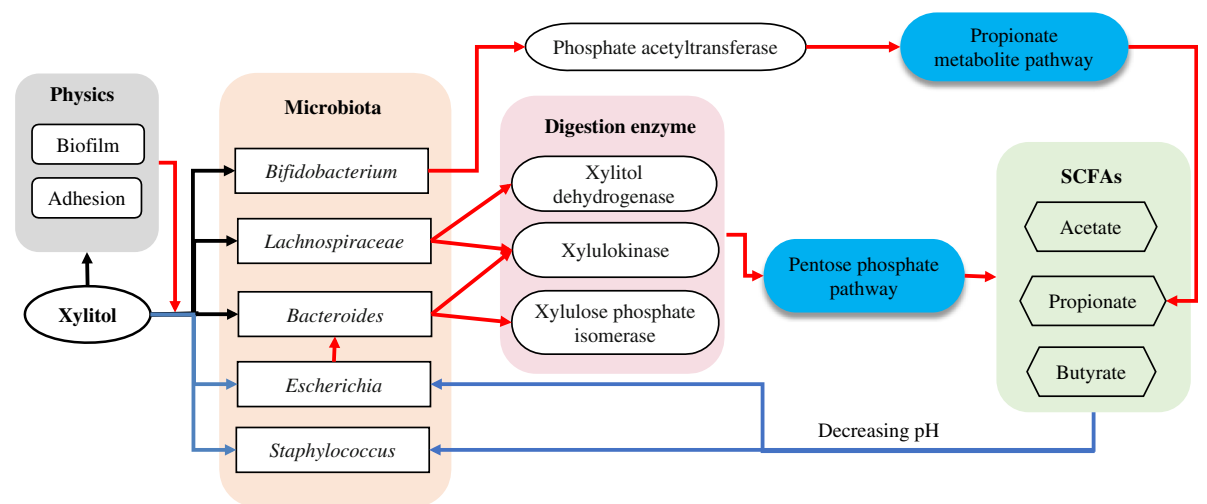

Fig. 6 The overall mechanism of xylitol prebiotic effect (red line_-promote, blue line—inhibit, black line—-neutral)

in the mucosa was varied with that in the lumen. The spatial structure of microbiota is a determining factor for cooperation and can drive the dynamics of microbiota [31, 58, 59]. Researchers had also reported that successful growth of the coculture experiments of Bacillus subtilis and the vitamin $\mathrm{B}_{1}$ auxotroph Serendipita indica was achieved only when these microorganisms were cultivated in a spatially organized environment that provides optimal conditions for cooperative interactions [31, 60]. In our study, the cross-feeding of xylitol was a benefit interaction not only for microbiota, but also for the host. In addition, we found that the xylitol obviously inhibited the biofilm formation and the adhesion to mucosa of toxin-producing $E$. coli but had no effects on beneficial bacteria like B. adolescentism (unpublished data). This can be a potential mechanism for prebiotic effects of xylitol.

In conclusion, we confirmed the effect of xylitol on propionate synthesis by combining the results of in vivo and in vitro experiments. The results of the metatranscriptome and metabolome provided macro-insights into propionate synthesis. At the same time, the potential mechanism of propionate synthesis was illustrated at the molecular level. In the food industry, we believe that xylitol can be used as a regulator to modulate and balance gut micro-ecology. The healthy and nutritional evaluation of functional foods should be performed on the basis of gut microbiota. We also proposed that the human gut is not only a large and complex fermentation system, but also an ecosystem.

\section{Conclusion}

In this study, the effect of xylitol on the gut microbiota and metabolic activities of mice was investigated by feeding mice xylitol. With the in vitro colon simulation system, the effects of xylitol on the dynamic changes in the microorganisms in the ascending, transverse, and descending colon and its effects on gas and SCFAs production were studied. Key bacteria were selected for single cultivation and co-culture of two and three bacteria, and the pathway in which xylitol was used by microorganisms was investigated. The effects of xylitol on the regulation of microorganisms were also studied at the molecular level. The possible mechanism of xylitol for propionate synthesis was also explained. The mechanism can be concluded as follows (Fig. 6): xylitol maintained the relative amount of Bifidobacterium, Bacteroides, Lactobacillus, and Lachnospariace in vivo and promoted enzymes related to xylitol from those bacteria. Moreover, it increased all SCFAs, especially propionate, in the lumen and butyrate in the mucosa, with a shift in its corresponding bacteria in the gut in vitro. A cross-feeding relationship was observed among L. reuteri, B. fragilis, and E. coli in the utilization of xylitol. At the molecular level, we also revealed a crossfeeding relationship in the microbiome. Xylitol dehydrogenase (EC 1.1.1.14), xylulokinase (EC 2.7.1.17), and xylulose phosphate isomerase (EC 5.1.3.1) were key enzymes in xylitol metabolism and were present in Bacteroides and Lachnospiraceae. Therefore, they were considered fundamental bacteria in xylitol digestion. In addition, xylitol also affected the metabolic pathway of propionate, significantly promoting the transcriptional level of the propionate ratelimiting enzyme, phosphate acetyltransferase (EC 2.3.1.8), in Bifidobacterium. Only those enzymes engaged from different bacteria together can support the energy metabolism and growth of micro-ecology, but on the other hand, they also enhanced the content of propionate, which lowered $\mathrm{pH}$ to restrict the growth of Escherichia. Through the cross-feeding and competition between those cells, xylitol can dynamically balance the relative amount of gut microbiome, in order to promote the health of the host.

\section{Methods and materials \\ Animals}

Twenty-four male C57BL/6 wild-type mice were purchased from Slack Company (Shanghai China). The rats were housed under controlled environmental conditions (temperature $23 \pm 1{ }^{\circ} \mathrm{C}$; humidity $55 \pm 5 \%$; 12-h light/ 
dark cycle) to adapt for 2 weeks, with a commercial food diet and water freely available. Animal experiments were carried out according to institutional guidelines for the care and the use of laboratory animals and approved by the animal ethics committee of China national institute of occupational health and poison control.

\section{Xylitol treatment}

After the adaption period, the C57BL/6 mice were randomly divided into 3 groups; 8 mice in each group were randomly divided into 3 cages and raised for 3 months. The control group was fed with AIN93M, while the experimental groups were fed with AIN93M supplemented with $2 \%$ or $5 \%$ xylitol $(\mathrm{w} / \mathrm{w})$, i.e., $2.17 \mathrm{~g} / \mathrm{kg} /$ day or $5.42 \mathrm{~g} /$ $\mathrm{kg} /$ day, respectively. According to the FDA introduction of clinical trials (Guidance for Industry Estimating the Maximum Safe Starting Dose in Initial Clinical Trials for Therapeutics in Adult Healthy Volunteers), xylitol supplementation is equal to $0.18 \mathrm{~g} / \mathrm{kg} /$ day and $0.44 \mathrm{~g} / \mathrm{kg} /$ day for a human being, which means estimated $11 \mathrm{~g}$ per day and $26 \mathrm{~g}$ per day for a $60-\mathrm{kg}$ adult. Water was freely available. Mice were weighed at day 0 and then monthly until sacrifice. Feces were collected monthly and were stored at $-80^{\circ} \mathrm{C}$ until DNA extraction and SCFA determination. After the mice were sacrificed, the brain, pancreas, colon, and liver of each animal were weighed by analytical balance, and the colon length was measured.

\section{In vitro three-stage colon simulation}

In vitro colon fermentation was conducted in Changdao Moni simulation system (CDMN) at Zhejiang Gongshang University as we described previously with some modification [61]. Three vessels (simulating ascending colon, transverse colon, descending colon) with volumes $300 \mathrm{~mL}, 400 \mathrm{~mL}$, and $300 \mathrm{~mL}$ are connected. Fecal microorganisms were inoculated into three vessels with $10 \%$ of vessel volume. Each vessel was then supplied with 15 mucosal beads ( $2 \mathrm{~g}$ agar was dissolved in $100 \mathrm{~mL}$ of distilled water by heating. After the solution was clear and transparent, it cooled to $60 \pm 5^{\circ} \mathrm{C}$. Weigh $0.5 \mathrm{~g}$ of mucin to dissolve and adjust the $\mathrm{pH}$ to about 6.8. After exposure to UV for $30 \mathrm{~min}, 7-8-\mathrm{mm}$ mucosal beads were obtained in the spherical abrasive tool). CDMN was automatically supplemented with $0.5 \mathrm{~mol} / \mathrm{L} \mathrm{NaOH}$ solution and diluted $\mathrm{HCl}$ to adjust the $\mathrm{pH}$. The temperature was kept at $37^{\circ} \mathrm{C}$ with the heating and cooling system. Anaerobic conditions were generated by flushing the headspace of all reactions and medium vessels with $\mathrm{N}_{2}$ for 30 min three times per day. After $24 \mathrm{~h}$ of inoculation and culture, in order to maintain the normal growth of microorganisms, feed and drain $300 \mathrm{~mL}$ of feed was supplied with the same volume waste to maintain the fermentation volume. Three mucosal beads in the vessels were replaced by new ones every day to simulate the mucosal regeneration of gut microbiota. After continuous inoculation for about 1 week, the microorganisms in the fermenter will stabilize (stage 1). Sterile xylitol was added to the feed, and it was ingested at a final concentration of $3 \%$ (equal to $0.27 \mathrm{~g} / \mathrm{kg} /$ day for a human being) to simulate xylitol digestion, which was calculated according to Livesey et al. [23] and Poeker et al. [39]. As $60 \%$ of xylitol can enter the colon, $3 \%$ of supplementation of xylitol into the simulation system is calculated for an estimated daily intake of $0.27 \mathrm{~g} / \mathrm{kg} /$ day for a $60-\mathrm{kg}$ adult (estimated $16 \mathrm{~g} /$ day), accounting for two reactors volume of $0.7 \mathrm{~L}$ compared to $0.75 \mathrm{~L}$ for the proximal colon volume (ascending colon, transverse colon), giving a mean retention time of $24 \mathrm{~h}$. The fermentation was stopped after 7 days (stage 2). Collect the fermentation broth and mucosal beads of each colon at each day of stage 2 and store the samples at $4{ }^{\circ} \mathrm{C}$ for use.

\section{DNA extraction and PCR amplification}

Bacterial DNA was extracted from 0.3-g feces, 1-mL fermentation broth, and one bead by using the TIANamp Bacteria DNA Kit (DP302-02, TIANGEN) according to the manufacturer's protocols. The V4-V5 region of the bacterial $16 \mathrm{~S}$ ribosomal RNA gene was amplified by PCR $\left(95^{\circ} \mathrm{C}\right.$ for $2 \mathrm{~min}$; followed by 25 cycles at $95^{\circ} \mathrm{C}$ for $30 \mathrm{~s}$, $55^{\circ} \mathrm{C}$ for $30 \mathrm{~s}$, and $72{ }^{\circ} \mathrm{C}$ for $30 \mathrm{~s}$; and a final extension at $72^{\circ} \mathrm{C}$ for $5 \mathrm{~min}$ ) using primers 338F $5^{\prime}$-ACTCCTACGG GAGGCAGCA-3' and 806R 5'-GGACTACHVGGG TWTCTAAT-3'. PCRs were performed in a $20-\mu \mathrm{L}$ mixture in triplicate and contained $4 \mu \mathrm{L}$ of $5 \times$ FastPfu Buffer, $2 \mu \mathrm{L}$ of $2.5 \mathrm{mM}$ dNTPs, $0.8 \mu \mathrm{L}$ of each primer $(5 \mu \mathrm{M}), 0.4 \mu \mathrm{L}$ of FastPfu Polymerase, and $10 \mathrm{ng}$ of template DNA.

Fungal DNA was extracted from $1 \mathrm{~mL}$ broth using the Plant Genomic DNA Kit (DP305-02, TIANGEN) according to the manufacturer's protocols. The ITS2 region of the fungal ITS gene was amplified by PCR $\left(98^{\circ} \mathrm{C}\right.$ for 30 s; followed by 35 cycles at $98^{\circ} \mathrm{C}$ for $10 \mathrm{~s}, 54^{\circ} \mathrm{C}$ for $30 \mathrm{~s}$, and $72{ }^{\circ} \mathrm{C}$ for $450 \mathrm{~s}$; and a final extension at $72{ }^{\circ} \mathrm{C}$ for 10 min) using primers fITS7: $5^{\prime}$-GTGARTCATCGAATCT TTG-3' and ITS4: 5'-TCCTCCGCTTATTGATATGC$3^{\prime}$. PCRs were performed in a $25-\mu \mathrm{L}$ mixture in triplicate and contained $12.5 \mu \mathrm{L}$ of $2 \times$ Phusion $^{\circ}$ Hot Start Flex Master Mix, $2.5 \mu \mathrm{L}$ of each primer $(1 \mu \mathrm{M})$, and $10 \mathrm{ng}$ of template DNA.

\section{Illumina MiSeq sequencing}

Amplicons were extracted from 2\% agarose gels and purified using the AxyPrep DNA Gel Extraction Kit (Axygen Biosciences, Union City, CA, USA) according to the manufacturer's instructions and quantified using QuantiFluor $^{\mathrm{Tm}}$-ST (Promega, USA). Purified amplicons were pooled in equimolar proportions and paired-end sequenced $(2 \times 300)$ on an Illumina MiSeq at Mingke 
Biotechnology (Hangzhou) Co., Ltd., China. The raw reads were deposited into the NCBI Sequence Read Archive (SRA) database.

\section{Processing of Illumina MiSeq sequencings data}

Raw fastq files were demultiplexed and quality-filtered using QIIME (version 1.17) with the following criteria: (i) the 250-bp reads were truncated at any site receiving an average quality score $<20$ over a 10-bp sliding window, discarding the truncated reads that were shorter than $50 \mathrm{bp}$; (ii) exact barcode matches, 2 nucleotide mismatch in primer matches, and reads containing ambiguous bases were removed; and (iii) only sequences with overlap longer than $10 \mathrm{bp}$ were assembled according to their overlap sequence. Reads that could not be assembled were discarded.

Operational units (OTUs) were clustered with a 97\% similarity cut-off using UPARSE (version $7.1 \mathrm{http}: / /$ drive5.com/uparse/), and chimeric sequences were identified and removed using UCHIME. The phylogenetic affiliation of each 16S rRNA gene sequence was analyzed by RDP Classifier (http://rdp.cme.msu.edu/) against the Silva (SSU115) 16S rRNA database using a confidence threshold of $70 \%$. The phylogenetic affiliation of each ITS2 gene was analyzed by RDP Classifier (http://rdp. cme.msu.edu/) against the Unite database.

\section{Determination of SCFAs}

0.3-g sample of pretreated feces from rats (supernatant from the mixture of feces after grinding with $1 \mathrm{~mL}$ ultrapure water after centrifugation at $6000 \mathrm{rpm}$ for $10 \mathrm{~min}$ ) or $1 \mathrm{~mL}$ of fermentation broth mixed with $100 \mu \mathrm{L}$ concentrated hydrochloric acid and $5 \mathrm{~mL}$ of ethyl ether was used. After extraction at room temperature for $20 \mathrm{~min}$, the mixture was centrifuged at $5000 \mathrm{rpm}$ for $10 \mathrm{~min}$ at $4{ }^{\circ} \mathrm{C}$. The top layer was removed to clean the tube and mixed with $500 \mu \mathrm{L}$ of $1 \mathrm{M} \mathrm{NaOH}$. After the second extraction and centrifugation, the water phase in the bottom layer was collected and removed to another tube. The bottom layer was filtered with a $0.22-\mu \mathrm{m}$ filter after adding $100 \mu \mathrm{L}$ concentrated hydrochloric acid. The SCFAs in the aliquot were determined by HPLC. Column: ZORBAX SB-Aq $(4.6 \times 250 \mathrm{~mm} 5$-Micron); mobile phase $0.025 \%$ phosphoric acid solution $(\mathrm{pH}=2.8)$ : acetonitrile $=95: 5$, flow rate $1.0 \mathrm{~mL} / \mathrm{min}$; injection volume $20 \mu \mathrm{L}$; detection wavelength $210 \mathrm{~nm}$; column temperature $30^{\circ} \mathrm{C}$ [62].

\section{Metatranscriptome sequencing RNA isolation, purification, and quantification}

Total RNA was isolated and purified using TRIzol reagent (Invitrogen, Carlsbad, CA, USA) following the manufacturer's procedure. The RNA amount and purity of each sample were quantified using a NanoDrop ND-1000 (NanoDrop, Wilmington, DE, USA). The RNA integrity was assessed by Agilent 2100 with a RIN number > 7.0.

\section{cDNA library construction}

Approximately $0.5 \mathrm{~g}$ of total RNA was used to deplete ribosomal RNA according to the instructions of the RiboZero $^{\mathrm{Tm}}$ rRNA Removal Kit (Illumina, San Diego, USA). After removing ribosomal RNAs, the remaining RNAs were fragmented into small pieces using divalent cations under high temperatures. Then, the cleaved RNA fragments were reverse-transcribed to create cDNA, which was then used to synthesize U-labeled second-stranded DNAs with E. coli DNA polymerase I, RNase H, and dUTP. An A-base was then added to the blunt ends of each strand, preparing them for ligation to the indexed adapters. Each adapter contained a T-base overhang for ligating the adapter to the A-tailed fragmented DNA. Single- or dual-index adapters were ligated to the fragments, and size selection was performed with AMPureXP beads. After heat-labile UDG enzyme treatment of the U-labeled second-stranded DNAs, the ligated products were amplified by PCR under the following conditions: initial denaturation at $95^{\circ} \mathrm{C}$ for $3 \mathrm{~min} ; 8$ cycles of denaturation at $98^{\circ} \mathrm{C}$ for $15 \mathrm{~s}$, annealing at $60^{\circ} \mathrm{C}$ for $15 \mathrm{~s}$, and extension at $72{ }^{\circ} \mathrm{C}$ for $30 \mathrm{~s}$; and a final extension at $72{ }^{\circ} \mathrm{C}$ for $5 \mathrm{~min}$. The average insert size for the final cDNA library was $300 \mathrm{bp}$ ( $\pm 50 \mathrm{bp}$ ). Finally, we performed 150 -bp paired-end sequencing on an Illumina HiSeq 4000 (LC-Bio Technology Co., Ltd., Hangzhou, Zhejiang Province, China) following the vendor's recommended protocol.

\section{Processing of metatranscriptome sequencing data}

Raw sequencing reads were processed to obtain valid reads for further analysis. First, sequencing adapters were removed from sequencing reads using cutadapt v1.9. Second, low-quality reads were trimmed by fqtrim v0.95 using a sliding-window algorithm. Third, reads were aligned to the host genome using bowtie2 v2.2.0 to remove host contamination. Once qualityfiltered reads were obtained, they were de novo assembled to construct the metatranscriptome for each sample with Trinity v2.2.0. All metatranscriptome contigs of all samples were clustered by CD-HIT v4.6.1 to obtain unigenes. Unigene abundance for a certain sample was estimated by TPM based on the number of aligned reads by bowtie 2 v2.2.0. The lowest common ancestor taxonomy of unigenes was obtained by aligning them against the NCBI NR database by DIAMOND v 0.7.12. Similarly, the functional annotation (GO, KEGG, eggNOG, CAZy, CARD, PHI) of unigenes was obtained. Based on the taxonomic and functional annotation of unigenes and the abundance profile of unigenes, the differential analysis was carried out at each taxonomic, functional, or gene-wise level by Fisher's exact test (nonreplicated groups) or Kruskal-Wallis test (replicated groups). 


\section{Metabolomic profiling identification}

The collected samples were thawed on ice, and metabolites were extracted with $50 \%$ methanol. Briefly, $20 \mu \mathrm{L}$ of a sample was extracted with $120 \mu \mathrm{L}$ of precooled $50 \%$ methanol, vortexed for $1 \mathrm{~min}$, and incubated at room temperature for $10 \mathrm{~min}$; the extraction mixture was then stored overnight at $-20^{\circ} \mathrm{C}$. After centrifugation at $4000 \mathrm{~g}$ for $20 \mathrm{~min}$, the supernatants were transferred into new 96-well plates. The samples were stored at $-80^{\circ} \mathrm{C}$ prior to the LC-MS analysis. In addition, pooled QC samples were also prepared by combining $10 \mu \mathrm{L}$ of each extraction mixture.

All samples were acquired by the LC-MS system following the instructions of the instrument. First, all chromatographic separations were performed using an ultraperformance liquid chromatography (UPLC) system (SCIEX, UK). An ACQUITY UPLC T3 column (100 mm $\times 2.1 \mathrm{~mm}, 1.8 \mu \mathrm{m}$, Waters, UK) was used for the reversed-phase separation. The column oven was maintained at $35^{\circ} \mathrm{C}$. The flow rate was $0.4 \mathrm{~mL} / \mathrm{min}$, and the mobile phase consisted of solvent A (water, $0.1 \%$ formic acid) and solvent $B$ (acetonitrile, $0.1 \%$ formic acid). The gradient elution conditions were set as follows: $0 \sim 0.5$ min, $5 \%$ B; $0.5 \sim 7 \mathrm{~min}, 5$ to $100 \% \mathrm{~B}$; 7 8 min, $100 \% \mathrm{~B}$; $8 \sim 8.1 \mathrm{~min}, 100$ to $5 \% \mathrm{~B} ; 8.1 \sim 10 \mathrm{~min}, 5 \% \mathrm{~B}$. The injection volume for each sample was $4 \mu \mathrm{L}$.

A high-resolution tandem mass spectrometer TripleTOF5600plus (SCIEX, UK) was used to detect metabolites eluted from the column. The Q-TOF was operated in both positive and negative ion modes. The curtain gas was set at 30 PSI, the ion source gas1 was set at 60 PSI, the ion source gas2 was set at $60 \mathrm{PSI}$, and the interface heater temperature was $650^{\circ} \mathrm{C}$. In the positive ion mode, the ion spray voltage floating was set at $5000 \mathrm{~V}$. In the negative ion mode, the ions pray voltage floating was set at $-4500 \mathrm{~V}$. The mass spectrometry data were acquired in IDA mode. The TOF mass range was from 60 to $1200 \mathrm{Da}$. The survey scans were acquired in $150 \mathrm{~ms}$, and as many as 12 product ion scans were collected if a threshold of 100 counts per second (counts/s) was exceeded and a 1+ charge-state was detected. The total cycle time was fixed to $0.56 \mathrm{~s}$. Four times bins were summed for each scan at a pulse frequency value of 11 $\mathrm{kHz}$ through monitoring of the $40-\mathrm{GHz}$ multichannel TDC detector with four-anode/channel detection. Dynamic exclusion was set at $4 \mathrm{~s}$. During the acquisition, the mass accuracy was calibrated every 20 samples. Furthermore, to evaluate the stability of the LC-MS during the whole acquisition, a quality control sample (pool of all samples) was acquired after every 10 samples.

The acquired MS data pretreatments including peak picking, peak grouping, retention time correction, second peak grouping, and annotation of isotopes and adducts were performed using XCMS software. LC-MS raw data files were converted into mzXML format and then processed by the XCMS, CAMERA, and metaX toolbox implemented with the $\mathrm{R}$ software. Each ion was identified by combining retention time (RT) and $\mathrm{m} / \mathrm{z}$ data. Intensities of each peak were recorded and a threedimensional matrix containing arbitrarily assigned peak indices (retention time- $m / z$ pairs), sample names (observations), and ion intensity information (variables) was generated.

The online KEGG, HMDB database was used to annotate the metabolites by matching the exact molecular mass data $(\mathrm{m} / \mathrm{z})$ of samples with those from the database. If a mass difference between observed and the database value was less than $10 \mathrm{ppm}$, the metabolite would be annotated and the molecular formula of metabolites would further be identified and validated by the isotopic distribution measurements. We also used inhouse fragment spectrum library of metabolites to validate the metabolite identification.

The intensity of peak data was further pre-processed by metaX. Those features that were detected in less than $50 \%$ of QC samples or $80 \%$ of biological samples were removed, the remaining peaks with missing values were imputed with the k-nearest neighbor algorithm to further improve the data quality. PCA was performed for outlier detection and batch effects evaluation using the pre-processed dataset. Quality control-based robust LOESS signal correction was fitted to the QC data with respect to the order of injection to minimize signal intensity drift over time. In addition, the relative standard deviations of the metabolic features were calculated across all QC samples, and those > 30\% were then removed.

Student $t$ tests were conducted to detect differences in metabolite concentrations between 2 phenotypes. The $p$ value was adjusted for multiple tests using an FDR (Benjamini-Hochberg).

Supervised PLS-DA was conducted through metaX to discriminate the different variables between groups. The VIP value was calculated. A VIP cut-off value of 1.0 was used to select important features.

\section{Cultivation of single, two, and three bacteria to evaluate xylitol fermentation \\ Bacterial cultivation}

Saccharomyces cerevisiae S33 was inoculated into Yeast Peptone Dextrose medium (Hangzhou microbial reagents Co. LTD, China), Bifidobacterium adolescentis JYBA-16 and Lactobacillus reuteri DSM20058 were inoculated into MRS medium (Hangzhou microbial reagents Co. LTD, China), and Bacteroides fragilis BNCC 336948 and Escherichia coli CICC 10032 were inoculated in TSB and LB medium (Hangzhou microbial reagents Co. LTD, China), respectively. After growing, 
aliquots from each medium were inoculated on the corresponding agar medium to pick pure and single colonies. The single and pure colonies were pre-cultured into the corresponding medium for growth until $\mathrm{OD}_{600}$ reaches $0.6 \sim 0.8$. One hundred microliters of each pure bacterial suspension was cultured to $10-\mathrm{mL}$ GAM medium at $37^{\circ} \mathrm{C}$ until $\mathrm{OD}_{600}$ reaches $0.6 \sim 0.8$. GAM contains (/L) $10 \mathrm{~g}$ pancreatic casein peptone, $3 \mathrm{~g}$ soy protein, $5 \mathrm{~g}$ yeast extract, $2 \mathrm{~g}$ beef extract, $13.5 \mathrm{~g}$ digested serum, $1.2 \mathrm{~g}$ beef liver extract, $2.5 \mathrm{~g}$ potassium dihydrogen phosphate, $3 \mathrm{~g}$ sodium chloride, $0.3 \mathrm{~g}$ cysteine- $\mathrm{HCl}$, $0.15 \mathrm{~g}$ sodium thioglycolate, $2 \mathrm{~g}$ glucose, and $0.3 \mathrm{~g}$ soluble starch. The $\mathrm{pH}$ was adjusted to $7.3 \pm 0.1$. B.adolescentis, L.reuteri, and B.fragilis were incubated into anaerobic condition, while $S$. cerevisiae and E.coli grown aerobically with $150 \mathrm{rpm}$ shaking.

A total of $100 \mu \mathrm{L}$ of bacterial suspension from the cultured GAM medium was added to the fresh GAM medium, and the number of bacteria was counted every $6 \mathrm{~h}$ until the concentration reached $10^{9} \mathrm{cell} / \mathrm{mL}$ by hemocytometer. Then, the suspension was diluted to $1 \times$ $10^{9} \mathrm{cell} / \mathrm{mL}$ by saline to guarantee the concentration of each bacterial inoculum for xylitol fermentation is the same.

\section{Xylitol fermentation}

The media of GAM with glucose and soluble starch depletion are named non-carbohydrate GAM media. The composition of GAM + glucose, GAM + xylitol, and combination of GAM media are non-carbohydrate GAM media with respective addition of $20 \mathrm{~g} / \mathrm{L}$ glucose, $20 \mathrm{~g} / \mathrm{L}$ xylitol, and $10 \mathrm{~g} / \mathrm{L}$ xylitol $+10 \mathrm{~g} / \mathrm{L}$ glucose. A total of $100 \mu \mathrm{L}$ of each diluted bacterial suspension from GAM medium (the concentration of diluted bacterial suspension was $1 \times 10^{9} \mathrm{cell} / \mathrm{mL}$ ) was inoculated at $100 \mathrm{~mL}$ of GAM + glucose medium, GAM + xylitol medium, a combination of GAM media, and non-carbohydrate GAM medium, for single-strain fermentation.

$100 \mu \mathrm{L}$ of diluted $E$. coli and $L$. reuteri suspension were co-cultured in $100 \mathrm{~mL}$ of GAM + glucose and GAM + xylitol media for double-strain fermentation. One hundred microliters of diluted E. coli, L. reuteri, and B. fragilis was transferred to $100 \mathrm{~mL}$ of GAM + glucose and GAM + xylitol media for three-strain fermentation. The fermentation lasted for $48 \sim 60 \mathrm{~h}$ under anaerobic condition. Two milliliters of aliquots from each medium was sampled every $2-4 \mathrm{~h}$ and diluted from $10^{0}$ to $10^{-7}$ for plating and counting. The sampled fermentation broth of E. coli, L. reuteri, B. fragilis, S. cerevisiae, and B. adolescentis were respectively plated into Maconkey Agar media, Chalmers Agarmedia, Modified GAM Agar media, Li-Mupirocin and Cysteine hydrochloride modified MRS media, and Yeast Peptone Dextrose Agar media and MRS for enumeration.
Maconkey Agar media, Modified GAM Agar media, and Li-Mupirocin and Cysteine hydrochloride modified MRS media were purchased from Qingdao Haibo Biotechnology Co. LTD, China. Chalmers Agarmedia, Yeast Peptone Dextrose Agar media, and MRS were purchased from Hangzhou microbial reagents Co. LTD, China.

\section{Statistical analyses}

SCFAs and microorganisms were analyzed by CCA (Canoco 5). The correlation between microbes was calculated by Spearman correlation with SPSS, and the correlation coefficient of correlation coefficient greater than 0.2 was selected and plotted with Cytoscape (v3.4.0). All data are shown as the mean \pm SD. The data were analyzed with ANOVA and Duncan's test for multiple comparisons with SPSS ver. 17.0. A value of $p<0.05$ was considered significant. FDR analysis of Benjamini and Hochberg (BH) method was applied to reduce the false positive rate for the high amount of data and a value of $Q<0.05$ was considered significant after FDR analysis. The optimal distribution was analyzed by @risk v7.5.0.1.

\section{Supplementary Information}

The online version contains supplementary material available at https://doi. org/10.1186/s40168-021-01029-6.

\section{Additional file 1. Additional information. \\ Additional file 2: Figure S1. Gamma distribution of microbiome fitted} according to chi-square standard.

Additional file 3: Figure S2. Expon distribution of alpha-diversity fitted according to chi-square standard.

Additional file 4: Figure S3. Phylum analysis of relative abundance of bacteria in mice colon. (Con: control group; $2 \% X Y$-a cohort of mouse supplied with diet contains $2 \%$ of xylitol; $5 \% X Y$ - a cohort of mouse supplied with diet contains $5 \%$ of xylitol; the number means the month to feed the mice)

Additional file 5: Figure S4. Phylum analysis of relative abundance of bacteria in different parts of in vitro colon (A: bacteria in lumen, B: bacteria in mucosa. AC, TC, DC presented Ascending Colon, Transverse colon and descending colon, the number means the days for adding xylitol).

Additional file 6: Figure S5. Histogram of changes in gas proportions in each segment of the colon (AC, TC, DC presented Ascending Colon, Transverse colon and descending colon. The number means the days for adding xylitol)

Additional file 7: Figure S6. Effects of xylitol on propanoate metabolites (Red-up regulate, Blue-down regulate).

Additional file 8: Figure S7. Effects of xylitol on pentose and glucuronate interconversion metabolites in vitro (Redup regulate, Bluedown regulate).

Additional file 9: Figure S8. Effects of xylitol on propionate metabolic enzymes (Red-up regulate, Blue-down regulate).

\section{Acknowledgements}

The authors would like to thank Zhejiang Huakang Pharmaceutical Co., Ltd for the xylitol supplement.

Authors' contributions

$S X$ and $K Y$ drafted the manuscript. $X B, X F, Y S$, and $C L$ analyzed the metabolomics data. $Y Z, Y G$, and $Y Z$ performed the $16 S$ rRNA sequencing. HW 
and JX prepared the study diets. SX, JY, SL, and ML performed the in vitro colonic fermentation study. JC and YC developed and supervised the UPLCQTOF-MS method used in the study. ST and JH conducted the statistics study. XZ drafted the manuscript and supervised the work. All authors critically revised the manuscript. All authors read and approved the final manuscript.

\section{Funding}

This study was funded by the National Natural Science Foundation of China (31501452), Foundation of Food Science and Engineering, the most important Discipline of Zhejiang Province (JYTsp20141082), and Project of international communication for construction of the first ranked discipline of Zhejiang Gongshang University (2017SICR106).

\section{Availability of data and materials}

The 165 rRNA and ITS gene sequencing dataset generated in this study are stored in National Center for Biotechnology Information (NCBI, https://www. ncbi.nlm.nih.gov/) and the project no. is PRJNA604899, PRJNA604957, PRJNA605597.

\section{Ethics approval and consent to participate}

For the animal experiment, in vitro colonic fermentation where the volunteers had given written consent for the fecal samples, and the collection and handling of the samples were performed according to the guidelines of the animal ethics committee of China national institute of occupational health and poison control; the ethical inspection no. is EAWE2018-002.

\section{Consent for publication}

Not applicable.

\section{Competing interests}

The authors declare no competing interests.

\section{Author details}

${ }^{1}$ School of Food Science and Biotechnology, Zhejiang Gongshang University, Hangzhou 310018, China. ² Zhejiang Huakang Pharmaceutical Co., Ltd., Kaihua 324302, China. ${ }^{3}$ Nutrition and Health Research Institute, COFCO Ltd., Beijing 102209, China. ${ }^{4}$ School of Medicine, Hangzhou Normal University, Hangzhou 310018, China. ${ }^{5}$ Laboratory of Aging and Cancer Biology of Zhejiang Province, Hangzhou Normal University, Hangzhou 311121, China. ${ }^{6}$ Yangtze Delta Institute of Tsinghua University, Jiaxing 314000, China. ${ }^{7}$ School of Food Science and Technology, Jiangnan University, Wuxi 214122, China.

Received: 1 September 2020 Accepted: 5 February 2021

\section{Published online: 18 March 2021}

\section{References}

1. Ur-Rehman S, Mushtaq Z, Zahoor T, Jamil A, Murtaza MA. Xylitol: a review on bioproduction, application, health benefits, and related safety issues. Crit Rev Food Sci Nutr. 2015;55(11):1514-28.

2. H. DGEA: Xylose and xylitol. Human Animal Nutrition 1978, 32:96-122.

3. Perez-Bibbins B, Torrado-Agrasar A, Salgado JM, Mussatto SI, Dominguez JM. Xylitol production in immobilized cultures: a recent review. Crit Rev Biotechnol. 2016;36(4):691-704.

4. da Silva SS, Chandel AK. D-Xylitol; 2012.

5. Sooch YLBS. Xylitol, an emerging prebiotic: a review. IJAPBR. 2017;2(2):67-73.

6. Canfora EE, Jocken JW, Blaak EE. Short-chain fatty acids in control of body weight and insulin sensitivity. Nat Rev Endocrinol. 2015;40(4):552-4.

7. Uebanso T, Kano S, Yoshimoto A, Naito C, Shimohata T, Mawatari K, Takahashi A. Effects of consuming xylitol on gut microbiota and lipid metabolism in mice. Nutrients 2017:9(7):756.

8. Sato T, Kusuhara S, Yokoi W, Ito M, Miyazaki K. Prebiotic potential of Lsorbose and xylitol in promoting the growth and metabolic activity of specific butyrate-producing bacteria in human fecal culture. FEMS Microbiol Ecol 2017;93(1):fiw227.

9. Chunhua Z, Hongjun D, Yanping Z, Yin L: Discovery of potential genes contributing to the biosynthesis of short-chain fatty acids and lactate in gut microbiota from systematic investigation in E. coli. NPJ Biofilms Microbiomes 2019, 5:19.
10. Tamura M, Hoshi C, Hori S. Xylitol affects the intestinal microbiota and metabolism of daidzein in adult male mice. Int J Mol Sci. 2013;14(12):239934007.

11. Salminen S, Salminen E, Koivistoinen P, Bridges J, Marks V. Gut microflora interactions with xylitol in the mouse, rat and man. Food Chem Toxicol. 1985:23(11):985-90.

12. Uhari M, Tapiainen T, Kontiokari T. Xylitol in preventing acute otitis media. Vaccine. 2000;19(5):S144-7.

13. Uhari M, Kontiokari T, Niemelä M. A novel use of xylitol sugar in preventing acute otitis media. Pediatrics. 1998;102(4 Pt 1):879-84.

14. Ferreira AS, Silva-Paes-Leme AF, Raposo NR, da Silva SS. By passing microbial resistance: xylitol controls microorganisms growth by means of its antiadherence property. Curr Pharm Biotechnol. 2015;16(1):35-42.

15. Cécile B, Aurélie F, Noélie T. Effect of xylitol on an in vitro model of oral biofilm. Oral Health Prev Dent. 2008;6(4):337-41.

16. Loo CY, Mitrakul K, Voss IB, Hughes CV, Ganeshkumar N. Involvement of an inducible fructose phosphotransferase operon in Streptococcus gordonii biofilm formation. J Bacteriol. 2003;185(21):6241-54.

17. Lee HJ, Kim SC, Kim J, Do A, Han SY, Lee BD, Lee HH, Lee MC, Lee SH, Oh T. Synergistic inhibition of Streptococcal biofilm by ribose and xylitol. Arch Oral Biol. 2015:60(2):304-12.

18. Wei T, Chen W, Qi X. Study on xylitol improving mice's gastrointestinal functions. Food Industrial Technology. 2001:22(5):23-4.

19. Grembecka M. Sugar alcohols - their role in the modern world of sweeteners: a review. Eur Food Res Technol. 2015;241(1):1-14.

20. Makinen KK. Sugar alcohol sweeteners as alternatives to sugar with special consideration of xylitol. Med Princ Pract. 2011;20(4):303-20.

21. Granstrom TB, Izumori K, Leisola M. A rare sugar xylitol. Part II: biotechnological production and future applications of xylitol. Appl Microbiol Biotechnol. 2007;74(2):273-6.

22. Lang K. Xylitol, its metabolism and clinical use. Wien Klin Wochenschr. 1971; 49(5):233-45

23. Livesey $\mathrm{G}$. Health potential of polyols as sugar replacers, with emphasis on low glycaemic properties. Nutr Res Rev. 2003;16(2):163-91.

24. Kamat S, Gaikwad S, Ravi Kumar A, Gade WN. Xylitol production by Cyberlindnera (Williopsis) saturnus, a tropical mangrove yeast from xylose and corn cob hydrolysate. J Appl Microbiol. 2013;115(6):1357-67.

25. Su B, Wu M, Zhang Z, Lin J, Yang L. Efficient production of xylitol from hemicellulosic hydrolysate using engineered Escherichia coli. Metab Eng. 2015:31:112-22

26. Makelainen HS, Makivuokko HA, Salminen SJ, Rautonen NE, Ouwehand AC. The effects of polydextrose and xylitol on microbial community and activity in a 4-stage colon simulator. J Food Sci. 2007;72(5):M153-9.

27. Sasaki D, Sasaki K, Ikuta N, Yasuda T, Fukuda I, Kondo A, Osawa R. Low amounts of dietary fibre increasein vitroproduction of short-chain fatty acids without changing human colonic microbiota structure. Sci Rep 2018;8(1): 435.

28. Chambers ES, Byrne CS, Morrison DJ, Murphy KG, Preston T, Tedford C, Garcia-Perez I, Fountana S, Serrano-Contreras JI, Holmes E, et al. Dietary supplementation with inulin-propionate ester or inulin improves insulin sensitivity in adults with overweight and obesity with distinct effects on the gut microbiota, plasma metabolome and systemic inflammatory responses: a randomised cross-over trial. Gut. 2019:68(8):1430-8.

29. Marzorati M, Van de Wiele T. An advanced in vitro technology platform to study the mechanism of action of prebiotics and probiotics in the gastrointestinal tract. J Clin Gastroenterol 2016;50(2):S124-S125.

30. Koh A, De Vadder F, Kovatcheva-Datchary P, Backhed F. From dietary fiber to host physiology: short-chain fatty acids as key bacterial metabolites. Cell. 2016;165(6):1332-45.

31. Williams CF, Walton GE, Jiang L, Plummer S, Garaiova I, Gibson GR. Comparative analysis of intestinal tract models. Annu Rev Food Sci Technol. 2015:6:329-50.

32. Reichardt N, Duncan SH, Young P, Belenguer A, McWilliam Leitch C, Scott KP, Flint HJ, Louis P. Phylogenetic distribution of three pathways for propionate production within the human gut microbiota. ISME J. 2014;8(6): 1323-35.

33. Louis P, Flint HJ. Formation of propionate and butyrate by the human colonic microbiota. Environ Microbiol. 2017;19(1):29-41.

34. Tengele AY, Gart E, Wiesmann M, Kiliaan A. Propionic acid and not caproic acid, attenuates nonalcoholic steatohepatitis and improves (cerebro) vascular functions in obese Ldlr/.Leiden mice. FASEB J. 2020;34(7):9575-93. 
35. Chambers ES, Viardot A, Psichas A, Morrison DJ, Murphy KG, Zac-Varghese SE, MacDougall K, Preston T, Tedford C, Finlayson GS, et al. Effects of targeted delivery of propionate to the human colon on appetite regulation, body weight maintenance and adiposity in overweight adults. Gut. 2015; 64(11):1744-54.

36. Gibson GR, Fuller R. Aspects of in vitro and in vivo research approaches directed toward identifying probiotics and prebiotics for human use. J Nutr. 2000;130(2S Suppl):391S.

37. Jack DL, Turner MW. Anti-microbial activities of mannose-binding lectin. Biochem Soc Trans. 2003;31(4):753-7.

38. Candela M, Maccaferri S, Turroni S, Carnevali P, Brigidi P. Functional intestinal microbiome, new frontiers in prebiotic design. Int J Food Microbiol. 2010;140(2-3):93-101.

39. Poeker SA, Geirnaert A, Berchtold L, Greppi A, Krych L, Steinert RE, de Wouters T, Lacroix C. Understanding the prebiotic potential of different dietary fibers using an in vitro continuous adult fermentation model (PolyFermS). Sci Rep. 2018;8(1):4318.

40. Cláudio DO, Rodrigues F, Gonalves SM, Goldman GH, Cunha C. The cell biology of the Trichosporon-host interaction. Front Cell Infect Microbiol. 2017;7:118.

41. Bunesova $V$, Lacroix C, Schwab C. Mucin cross-feeding of infant Bifidobacteria and Eubacterium hallii. Microb Ecol. 2017;75(1):1-11.

42. Li J, Convertino M. Optimal microbiome networks: macroecology and criticality. Entropy 2019;21(5):506.

43. Löfblom J, Rosenstein R, Nguyen MT, Staehl S, Goetz F: Staphylococcus carnosus: from starter culture to protein engineering platform. Appl Microbiol Biotechnol. 2017;101:8293-07.

44. Hugenholtz F, de Vos WM. Mouse models for human intestinal microbiota research: a critical evaluation. CMLS. 2018;75(1):149-60.

45. Nguyen TLA, Vieira-Silva S, Liston A, Raes J. How informative is the mouse for human gut microbiota research? DMM. 2015;8(1):1-16.

46. Turroni F, Duranti S, Milani C, Lugli GA, van Sinderen D, Ventura M. Bifidobacterium bifidum: a key member of the early human gut microbiota. Microorganisms. 2019;7(11):544.

47. Marißen J, Haiß A, Meyer C, Van Rossum T, Bünte LM, Frommhold D, Gille C, Goedicke-Fritz S, Göpel W, Hudalla H, et al. Efficacy of Bifidobacterium longum, B. infantis and Lactobacillus acidophilus probiotics to prevent gut dysbiosis in preterm infants of $28+0-32+6$ weeks of gestation: a randomised, placebo-controlled, double-blind, multicentre trial: the PRIMAL Clinical Study protocol. BMJ Open. 2019;9(11):e032617.

48. Costalos C, Kapiki A, Apostolou M, Papathoma E. The effect of a prebiotic supplemented formula on growth and stool microbiology of term infants. Early Hum Dev. 2008;84(1):45-9.

49. Langlands SJ, Hopkins MJ, Coleman N, Cummings JH. Prebiotic carbohydrates modify the mucosa associated microflora of the human large bowel. Gut. 2004:53(11):1610-6.

50. Zengler K, Zaramela LS. The social network of microorganisms - how auxotrophies shape complex communities. Nat Rev Microbiol. 2018;16(6): 383-90.

51. de Theije CG, Bavelaar BM, Lopes da Silva S, Korte SM, Olivier B, Garssen J, Kraneveld AD. Food allergy and food-based therapies in neurodevelopmental disorders. Pediatr Allergy Immunol. 2014;25(3):218-26.

52. Meehan CJ, Beiko RG. A phylogenomic view of ecological specialization in the Lachnospiraceae, a family of digestive tract-associated bacteria. Genome Biol Evol. 2014;6(3):703-13.

53. Rowland I, Gibson G, Heinken A, Scott K, Swann J, Thiele I, Tuohy K. Gut microbiota functions: metabolism of nutrients and other food components. Eur J Nutr. 2018;57(1):1-24.

54. Naaber $\mathrm{P}$, Mikelsaar RH, Salminen S, Mikelsaar M. Bacterial translocation, intestinal microflora and morphological changes of intestinal mucosa in experimental models of Clostridium difficile infection. J Med Microbiol. 1998; 47(7):591-8

55. Assev S, Scheie AA. Xylitol metabolism in xylitol-sensitive and xylitolresistant strains of streptococci. Apmis. 2010;94B(1-6):239-43.

56. Hilgers EJA, Schöttler MA, Mettler-Altmann T, Krueger S, Dörmann P, Eicks M, Flügge U-I, Häusler RE. The combined loss of triose phosphate and xylulose 5-phosphate/phosphate translocators leads to severe growth retardation and impaired photosynthesis in Arabidopsis thaliana tpt/xpt double mutants. Front Plant Sci. 2018;9:1331.
57. Banfield MJ, Salvucci ME, Baker EN, Smith CA. Crystal structure of the NADP(H)-dependent ketose reductase from Bemisia argentifolii at $2.3 \mathrm{~A}$ resolution. J Mol Biol. 2001;306(2):239-50.

58. Han Q, Eiteman MA: Coupling xylitol dehydrogenase with NADH oxidase improves I-xylulose production in Escherichia coli culture. Enzyme Microb Technol 2017, 106(undefined):106-113.

59. Gudelj I, Kinnersley M, Rashkov P, Schmidt K, Rosenzweig F. Stability of cross-feeding polymorphisms in microbial communities. PLoS Comput Biol. 2016;12(12):e1005269.

60. Soleimani M, Tabil LG, Panigrahi S. Bio-production of a polyalcohol (xylitol) from lignocellulosic resources: a review. CSBE-SCGAB. 2006:06-106. https:// doi.org/10.13031/2013.22064

61. Xu Y, Xiang S, Ye K, Zheng Y, Feng X, Zhu X, Chen J, Chen Y. Cobalamin (vitamin B12) induced a shift in microbial composition and metabolic activity in an in vitro colon simulation. Front Microbiol. 2018;9:2780.

62. Wang C, Gao PX, Jing-Nan XU, Wen-Gong YU, Xin-Zhi LU. Establishment and application of extraction and determination method for short-chain fatty acids in feces of mice. Prog Mod Biomed. 2017;17(06):1012-32.

\section{Publisher's Note}

Springer Nature remains neutral with regard to jurisdictional claims in published maps and institutional affiliations.
Ready to submit your research? Choose BMC and benefit from:

- fast, convenient online submission

- thorough peer review by experienced researchers in your field

- rapid publication on acceptance

- support for research data, including large and complex data types

- gold Open Access which fosters wider collaboration and increased citations

- maximum visibility for your research: over $100 \mathrm{M}$ website views per year

At BMC, research is always in progress.

Learn more biomedcentral.com/submissions 